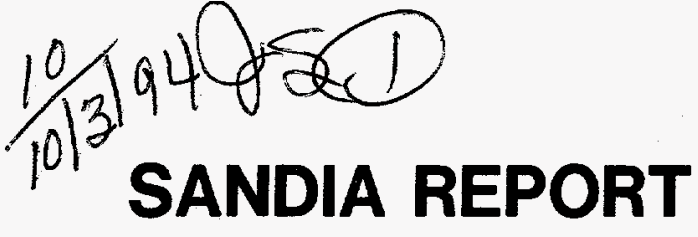

SAND94-0484 - UC-528

Unlimited Release

Printed March 1994

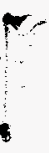

\title{
Reentry Safety for the Topaz II Space Reactor: Issues and Analyses
}

Leonard W. Connell, Larry C. Trost

\author{
Prepared by \\ Sandia National Laboratories \\ Albuquerque, New Mexico 87185 and Livermore, California 94550 \\ for the Unlted States Department of Energy \\ under Contract DE-AC04-94AL85000
}

Approved for public release; distribution is unlimited.

$+$

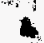

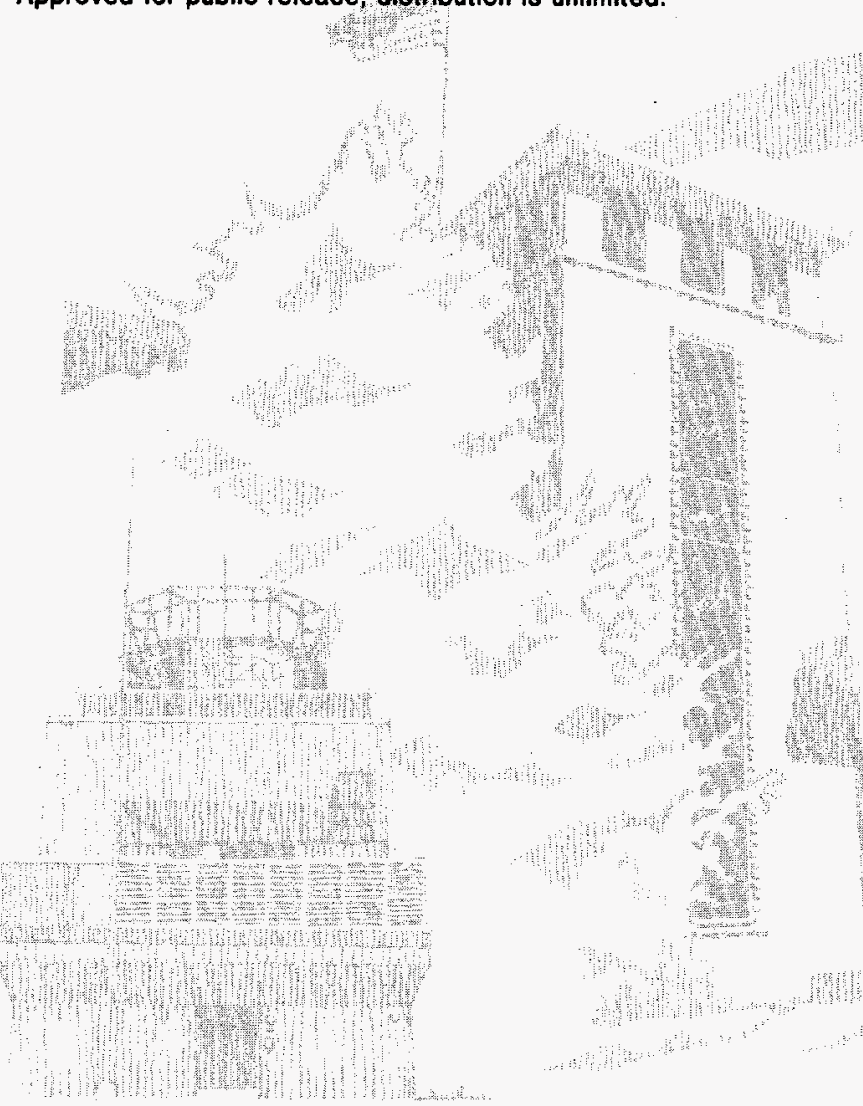




\section{DISCLAIMER}

This report was prepared as an account of work sponsored by an agency of the United States Government. Neither the United States Government nor any agency thereof, nor any of their employees, make any warranty, express or implied, or assumes any legal liability or responsibility for the accuracy, completeness, or usefulness of any information, apparatus, product, or process disclosed, or represents that its use would not infringe privately owned rights. Reference herein to any specific commercial product, process, or service by trade name, trademark, manufacturer, or otherwise does not necessarily constitute or imply its endorsement, recommendation, or favoring by the United States Government or any agency thereof. The views and opinions of authors expressed herein do not necessarily state or reflect those of the United States Government or any agency thereof. 


\section{DISCLAIMER}

Portions of this document may be illegible in electronic image products. Images are produced from the best available original document. 
SAND94-0484

Unlimited Release

Printed March 1994

\title{
REENTRY SAFETY FOR THE TOPAZ || SPACE REACTOR: ISSUES AND ANALYSES
}

\author{
Leonard W. Connell \\ and \\ Larry C. Trost \\ Strategic Studies Center \\ Sandia National Laboratories \\ Albuquerque, New Mexico 87185
}

\begin{abstract}
This report documents the reentry safety analyses conducted for the TOPAZ II Nuclear Electric Propulsion Space Test Program (NEPSTP). Scoping calculations were performed on the reentry aerothermal breakup and ground footprint of reactor core debris. The calculations were used to assess the risks associated with radiologically cold reentry accidents and to determine if constraints should be placed on the core configuration for such accidents. Three risk factors were considered: inadvertent criticality upon reentry impact, atmospheric dispersal of U-235 fuel, and the Special Nuclear Material Safeguards risks. Results indicate that the risks associated with cold reentry are very low regardless of the core configuration. Core configuration constraints were therefore not established for radiologically cold reentry accidents.
\end{abstract}




\section{Acknowledgments}

Many thanks to Don Potter and Channy Wong for the aerothermodynamics calculations, to Diane McCallum for the tech-art support, and to Richard Preston, Frank Thome, and Al Marshall for providing motivational support. 


\section{Table of Contents}

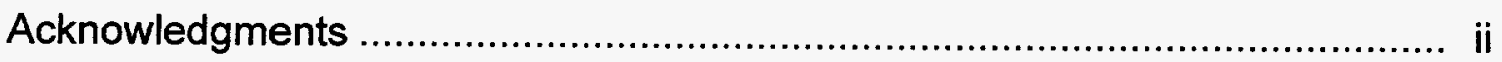

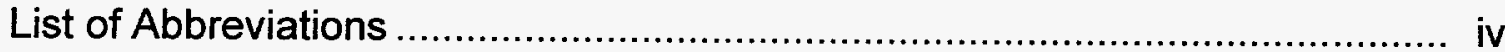

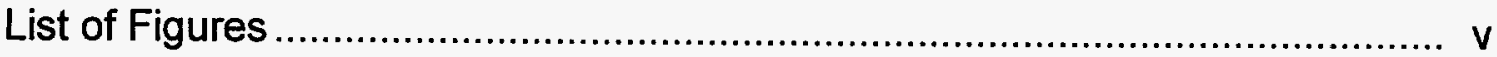

1. Introduction .............................................................................. 1

2. Reentry Safety Functional Requirements: Historical Perspective ......... 2

2.1. The Cosmos 954 Incident.................................................................. 3

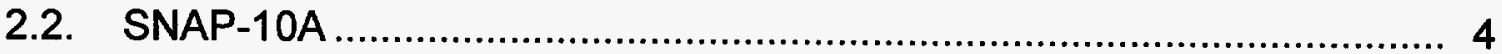

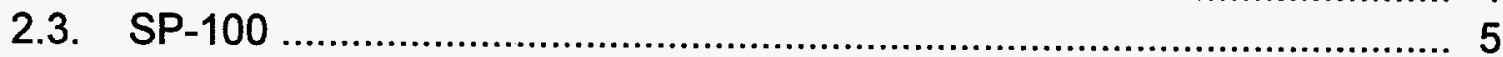

2.4. Summary of Historical Review......................................................... 7

3. Cold Reentry of TOPAZ II: Breakup Analysis..................................... 7

3.1. Analysis Assumptions and Methodology ........................................... 7

3.2. Aerodynamic Assumptions ................................................................ 8

3.3. Heating/Thermal Response Assumptions ........................................ 10

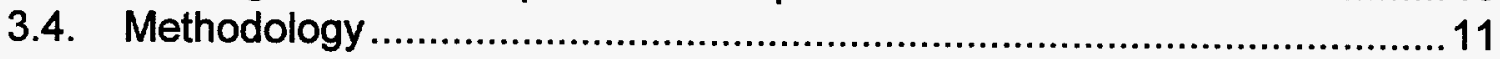

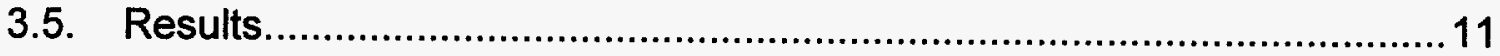

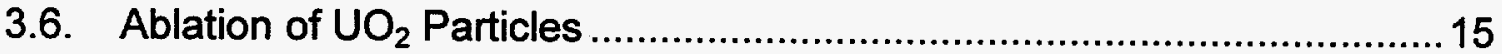

3.7. TOPAZ II Reentry Breakup Summary ................................................. 16

4. Analysis of the Need for Cold Reentry Core Configuration Constraints.. 16

4.1. Cold Reentry Radiological Safety Risks .............................................17

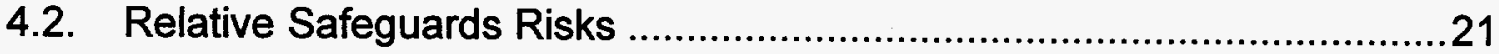

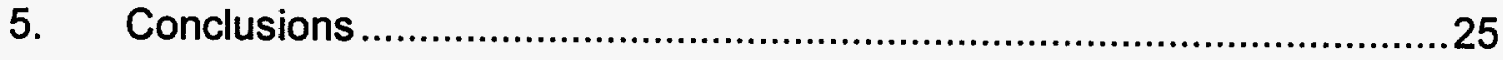




\section{List of Abbreviations}

\begin{tabular}{ll} 
DAC & Derived Air Concentration \\
DOD & Department of Defense \\
DOE & Department of Energy \\
Gy/hr & Gray per hour, 1 Gray = 1 Joule/kilogram radiation energy deposition \\
HEU & Highly Enriched Uranium \\
IAEA & International Atomic Energy Agency \\
km & kilometer \\
kWth & kilowatt-thermal \\
LiH & Lithium Hydride \\
MeV & Million Electron Volt \\
ml & milliliter \\
mm & millimeter \\
MW-sec & Mega-Watt-second \\
NaK & Sodium - Potassium \\
NASA & National Aeronautics and Space Administration \\
NEPSTP & Nuclear Electric Space Test Program \\
Pu & Plutonium \\
REM & Roentgen Equivalent Man, 1 REM = 100 erg/gm \\
RORSAT & Radar Ocean Reconnaissance Satellite \\
RTG & Radioisotope Thermoelectric Generator \\
RTS & Random Tumble and Spin \\
Rx & Reactor \\
SNAP & Space Nuclear Auxiliary Power \\
SNM & Special Nuclear Material \\
SP-100 & Space Power - 100 kilowatt-electric \\
T1/2 & Half-life for radioactive decay, Half-time for aerosol retention time \\
TEM & Thermo-Electric-Magnetic \\
TFE & Thermionic Fuel Element \\
TSAP & Trajectory Simulation and Analysis Program \\
U & Uranium \\
U-Mo & Uranium - Molybdenum alloy \\
$\mu$ Ci & micro-Curie, 1 Ci = 3.7E10 disintegrations per second \\
$\mu$ Um & micro-meter \\
UO 2 & Uranium dioxide \\
We & Watt-electric \\
ZrH & Zirconium Hydride \\
& \\
& \\
\hline
\end{tabular}




\section{List of Figures}

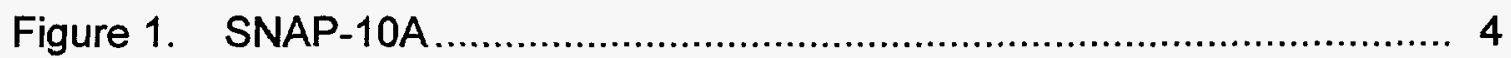

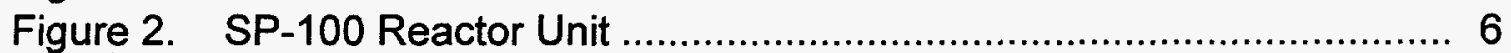

Figure 3. TOPAZ II System .................................................................. 8

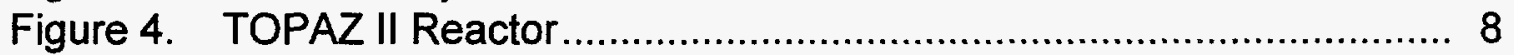

Figure 5. Reentry Breakup Assumptions ............................................... 9

Figure 6. Thermal Response of Reactor Support Legs ..............................11

Figure 7. Thermal Response of Reactor Vessel Top Section........................12

Figure 8. Thermal Response of Reactor Vessel Sidewall ...........................13

Figure 9. Thermal Response of Reactor Vessel Bottom Section ................... 14

Figure 10. TOPAZ II Breakup Sequence...................................................... 14

Figure 11. $\mathrm{UO}_{2}$ Pellet Ablation Parametric Analysis ................................... 15

Figure 12. Specific Activity of Enriched Uranium ........................................18

Figure 13. $\mathrm{UO}_{2}$ Aerosol Inhalation Pathways ............................................ 19

Figure 14. Los Alamos Calculations of SNM Diversion Probability..................22

Figure 15. TOPAZ II Fuel Rod Impact Points .............................................24

Figure 16. TOPAZ II Fuel Pellet Impact Points...............................................24 


\section{Introduction}

The accidental reentry of a TOPAZ II reactor introduces certain risk factors, the consequences of which depend on the radiological state of the reactor. For purposes of discussion, the term "cold" reactor will be used to describe one whose radioactivity is very low, approximately equal to the intrinsic activity of a virgin core. A cold reactor has either never been operated (i.e. never been taken critical) or, subsequent to power operation, has been maintained in a sub-critical state for a sufficient time to allow the fission and activation products to decay below the intrinsic level. In contrast, a "hot" reactor is defined as one whose radioactivity is much higher than the intrinsic level of the virgin reactor fuel. For a cold reactor, the radiological safety risks introduced by inadvertent reentry are due mainly to possible impact criticality accidents and possible dispersal and aerosolization of the virgin, highly enriched uranium (HEU) fuel. The safeguards risk is due to the potential for retrieval and weaponization of the HEU by unauthorized groups or states. The risks associated with radiologically hot reentry accidents include all the above plus the atmospheric and ground dispersal of radioactive fission products.

A principal factor bearing on reentry safety is the behavior of the reactor vessel and core materials following exposure to the intense reentry aerothermal heating environment. To minimize the safety risks, the ideal post-reentry-core-configuration would be either complete breakup and aerosolization at high altitude $(15$ to $20 \mathrm{~km})$ or alternatively, intact-through-impact survival. In the former case, the risk is minimized by diluting the radiation to well below background levels, although all humans are potentially exposed. In the latter case, risks are minimized by confining the radiation to a single impact point and limiting the number of humans receiving radiation exposure. This reentry safety concept was codified in the NEPSTP Reentry Core Configuration Safety Functional Requirement, which states: "For any credible radiologically hot reentry accident, the reactor core shall remain essentially intact or alternatively, shall undergo essentially complete dispersal of the radioactive materials at high altitude."

This is one of several reentry safety functional requirements established for NEPSTP, and reentry safety is but one of several subsets that comprise the complete set of safety functional requirements, covering all aspects of space nuclear reactors, including reactor startup, inadvertent criticality, operations in space, reentry, and safeguards. These requirements represent a code of conduct for the safe operation of space nuclear reactors as distilled from historical experience. Since they are not reactor or mission specific, the ways and means of compliance are not unique. These functional requirements will be used to establish mission operational safety procedures and to determine the extent of system modifications to be made to TOPAZ II.

Past U.S. space nuclear programs focused on the reentry safety of radiologically hot nuclear systems, such as Radioisotopic Thermoelectric Generators (RTGs) which use $\mathrm{Pu}-238$, or space nuclear reactors containing a fission product inventory due to past operation. As will be discussed, hot reentry is precluded from NEPSTP mission 
profiles. Therefore, in the case of NEPSTP, the Reentry Core-Configuration Safety Functional Requirement for hot reentry accidents is satisfied by ensuring that such accidents are not credible.

Cold reentry accidents, however, cannot be precluded and remain credible based on the failure rates of existing space launch vehicles. The reentry core-configuration constraint applied to hot reentry accidents was not extended to cold reentry because analysis of cold reentry demonstrated that the nuclear risk factors were very low, regardless of the core configuration. This report presents the analysis performed on cold reentry along with the issues that were considered in reaching the decision not to place core-configuration constraints on cold reentry accidents. Section 2 discusses the historical underpinnings of the NEPSTP Reentry Safety Functional Requirements. Section 3 presents results of the reentry breakup modeling performed on TOPAZ II and Section 4 discusses the implications of these results on the main nuclear risk factors for cold reentry: impact criticality, nuclear safeguards, and atmospheric dispersal of virgin HEU.

\section{Reentry Safety Functional Requirements: Historical Perspective}

The principal NEPSTP reentry safety functional requirements are:

1. For any credible radiologically hot reentry accident, the reactor shall remain essentially intact or alternatively, shall undergo essentially complete dispersal of the radioactive materials at high altitude.

2. The probability and consequences of an inadvertent reentry shall be made as low as reasonably achievable.

3. The reactor shall remain subcritical throughout an inadvertent reentry and earth impact.

Note that the Reentry Core-Configuration Safety Functional Requirement (No. 1 above), pertains only to hot reentry accidents. NEPSTP satisfied this with operational constraints and design modifications which make hot reentry accidents not credible. The initial orbital aititude for NEPSTP will exceed $1000 \mathrm{~km}$, resulting in an orbital lifetime of several thousand years, far greater than the time required for the fission and activation products to decay below the intrinsic level of radioactivity associated with virgin $\mathrm{HEU}$ and its actinide daughters. An anticriticality device will be retrofitted to TOPAZ II, preventing water immersion criticality accidents and precluding reactor startup until the stable operating orbit is verified and the device is deactivated. The development of these requirements was influenced by past space reactor programs.

A better understanding of the NEPSTP reentry safety requirements will, therefore, be obtained by reviewing the history of space nuclear safety, including the reentry accident involving the Russian space reactor Cosmos 954. This section also compares and contrasts the NEPSTP reentry safety program with those of SNAP-10A and SP-100. 


\subsection{The Cosmos 954 Incident}

The Cosmos 954 nuclear reactor-powered satellite was launched by the Soviet Union on September 18, 1977. On January 24, 1978 the satellite, including the reactor, reentered the earth's atmosphere over northern Canada leaving a debris footprint extending $1000 \mathrm{~km}$ to the northeast from Great Slave Lake to Baker Lake with an average width of $48 \mathrm{~km}$. Cosmos 954 belonged to a series of satellites called Radar Ocean Reconnaissance SATellites (RORSATs) which performed a military mission. The RORSATS were operated in low earth orbit where they would function for several months before the orbit became unstable due to atmospheric drag. Prior to reentry, the reactor was designed to separate from the spacecraft and transfer to a higher orbit where it would remain for a minimum of 500 years. The reactor aboard Cosmos 954 failed to separate for reasons that are unknown, and reentered the atmosphere along with the entire spacecraft.

Details of the design of the Cosmos 954 satellite and reactor were never made public by the Soviet authorities. Open literature publications (Bennett 1989) by U.S. experts have provided some details about the system based partly on speculation from the debris collected and partly from the scant information available on the Soviet space nuclear reactor program. In addition, a recent publication by Russian scientists has filled in some of the gaps. The reactor contained approximately $50 \mathrm{~kg}$ of a uraniummolybdenum alloy fuel with a 90 percent enrichment in U-235. The RORSAT reactors operated on fast neutrons and generated approximately $100 \mathrm{~kW}$ of thermal power. The core was arranged into 37 cylindrical fuel elements clad in stainless steel or niobium. The reactor vessel measured approximately $50 \mathrm{~cm}$ in length and $25 \mathrm{~cm}$ in diameter and was constructed of stainless steel. An electromagnetic pump transferred NaK coolant from the core to thermoelectric converters which generated approximately $5 \mathrm{kw}$ of electrical power. Reactor control was maintained by 6 in/out control rods composed of boron carbide with beryllium followers used for radial reflectors. The mass of the reactor was estimated to be less than $390 \mathrm{~kg}$ while the entire spacecraft mass was approximately $4000 \mathrm{~kg}$.

Shortly after reentry, the Canadian and U.S. Nuclear Emergency Search Teams assembled and began to structure a joint recovery program, code named Operation Morning Light (DOE 1978). The teams searched approximately 120,000 square kilometers of the Northwest Territories and were able to recover about $65 \mathrm{~kg}$ of debris. Debris contact dose rates ranged from approximately $5 \mathrm{~Gy} / \mathrm{hr}$ for some of the larger fragments to $0.1 \mathrm{mGy} / \mathrm{hr}$ for the small fuel particles. Contamination of air, water, and food supplies was not detected. An estimated $\$ 12$ million was spent by Canada and $\$ 2.7$ million by the U.S. for the cleanup which ended in October of 1978 . Less than 0.1 percent of the fuel was recovered. The remaining particles were assumed to have been dispersed world-wide by high altitude winds. They were not considered to pose a serious radiological hazard due to their low dose rates and insolubility in water. The break-up of the uranium-molybdenum fuel into an aerosol was predicted by Russian investigators (Koroteev et al. 1991) and confirmed by the findings of the Morning Light 
Team. Dispersal and disintegration of the fuel was, in fact, part of the reentry safety design of Cosmos 954. However, the survival of other radioactive components, such as the "antlers" (assumed to be the control rods) indicates that the policy of complete dispersal and aerosolization is difficult to achieve.

Although similarities exist between the Cosmos 954 and TOPAZ II reactors, fundamental differences make it difficult to draw meaningful conclusions about the reentry behavior of TOPAZ II from knowledge of the reentry of Cosmos 954. Most significant among the many differences is the fuel. The melt temperature of U-Mo is practically the same as uranium metal, $1403 \mathrm{~K}$, while $\mathrm{UO}_{2}$, a ceramic, has a melt temperature of $3150 \mathrm{~K}$, making it more resistant to melt-ablation during reentry. Additionally, the structural design of the TOPAZ II reactor is much more robust than the RORSAT design.

The Cosmos 954 accident made manifest the problems inherent with hot operations in low earth orbit. The short lifetime orbit permitted a hot reentry and the increased radiological risks associated with it relative to cold reentry. The accident also revealed the great difficulty of obtaining complete dispersal of the hot debris.

\subsection{SNAP-10A}

The SNAP-10A space reactor, shown below in Figure 1, was placed into a $1300 \mathrm{~km}$ circular polar orbit by an Atlas-Agena launch vehicle on April 3, 1965, becoming the first nuclear reactor in space and the only US space reactor to be launched.

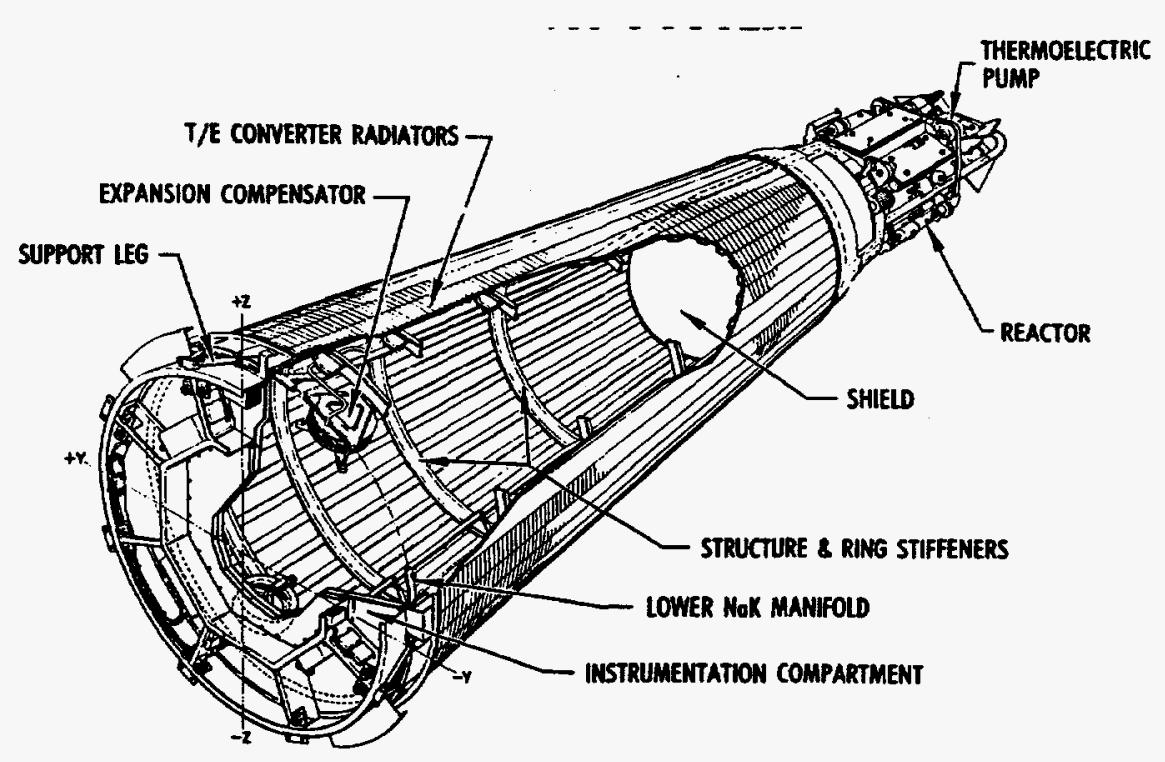

Figure 1. SNAP-10A 
At this altitude, SNAP-10A will remain in orbit for approximately 3800 years. SNAP-10A is a direct thermoelectric conversion system weighing $440 \mathrm{~kg}$ and measuring $3.4 \mathrm{~m}$ in length and $1.3 \mathrm{~m}$ base diameter, and 7.5 degree cone half-angle. The fission heat from the reactor is transported to the thermoelectric conversion system via NaK liquid metal coolant with flow maintained by a small thermoelectric pump. The epithermal reactor contains approximately $5 \mathrm{~kg}$ of $\mathrm{U}-235(93 \%)$ alloyed with zirconium and hydrated to near saturation and was rated at $34 \mathrm{kWth}$ for one year.

SNAP-10A employs operational constraints to preclude the reentry of a hot reactor system. An orbital altitude of $1300 \mathrm{~km}$ was chosen, which has a sufficiently long lifetime to permit the activity of the fission and activation products built up over the operating time of the reactor to decay down to a level less than or equal to the actinides. In addition, start-up of the reactor was not permitted until confirmation that the stable orbit had been achieved. Under these restrictions, reentry accidents occurring during launch and at the end of orbital life do not pose a serious radiological hazard since, in both cases, the radioactivity of the system is very low. These same constraints are observed by NEPSTP.

A thorough examination of the reentry heating and break-up of SNAP-10A was performed at both the analytical and experimental levels, including a reentry test of a full scale model (Elliott 1963). The conclusion drawn from this extensive program was that the reactor vessel would melt and release the core materials. Uncertainties existed, however, as to the fate of the fuel elements and complete ablation and dispersal of the fuel could not be guaranteed. Although complete dispersal of the core was the desired objective, no attempts were made to actively disperse the core, such as with an explosive charge. Calculations demonstrated that the risks to the public from reentry accidents are very low. Intact reentry through the use of a heat shield is not discussed in the SNAP-10A documents. The operational constraints on orbital lifetime and start-up were the key elements in establishing reentry safety. Analyses and tests also demonstrated that the reactor could become super-critical upon immersion in water and undergo a prompt disassembly with a $70 \mathrm{MW}$-sec energy yield. Due to the low probability of a launch abort-water immersion accident occurring with humans in the nearby vicinity, the SNAP-10A safety team opted to dispense with anti-criticality safety mechanisms during launch.

\subsection{SP-100}

The SP-100 space reactor, shown in Figure 2, has been under development since the early 1980 s as a joint DOE, DOD, NASA, interagency effort. Budget constraints and the absence of a user have caused the termination of the program. SP-100 is a fast reactor with uranium nitride fuel and liquid metal coolant. The major subsystems include a beryllium sliding reflector, radiation shield, primary heat transport system (lithium liquid metal), thermoelectric electro-magnetic (TEM) pumps, thermoelectric power converters, heat pipe radiators, and a carbon-carbon reentry heat shield. 


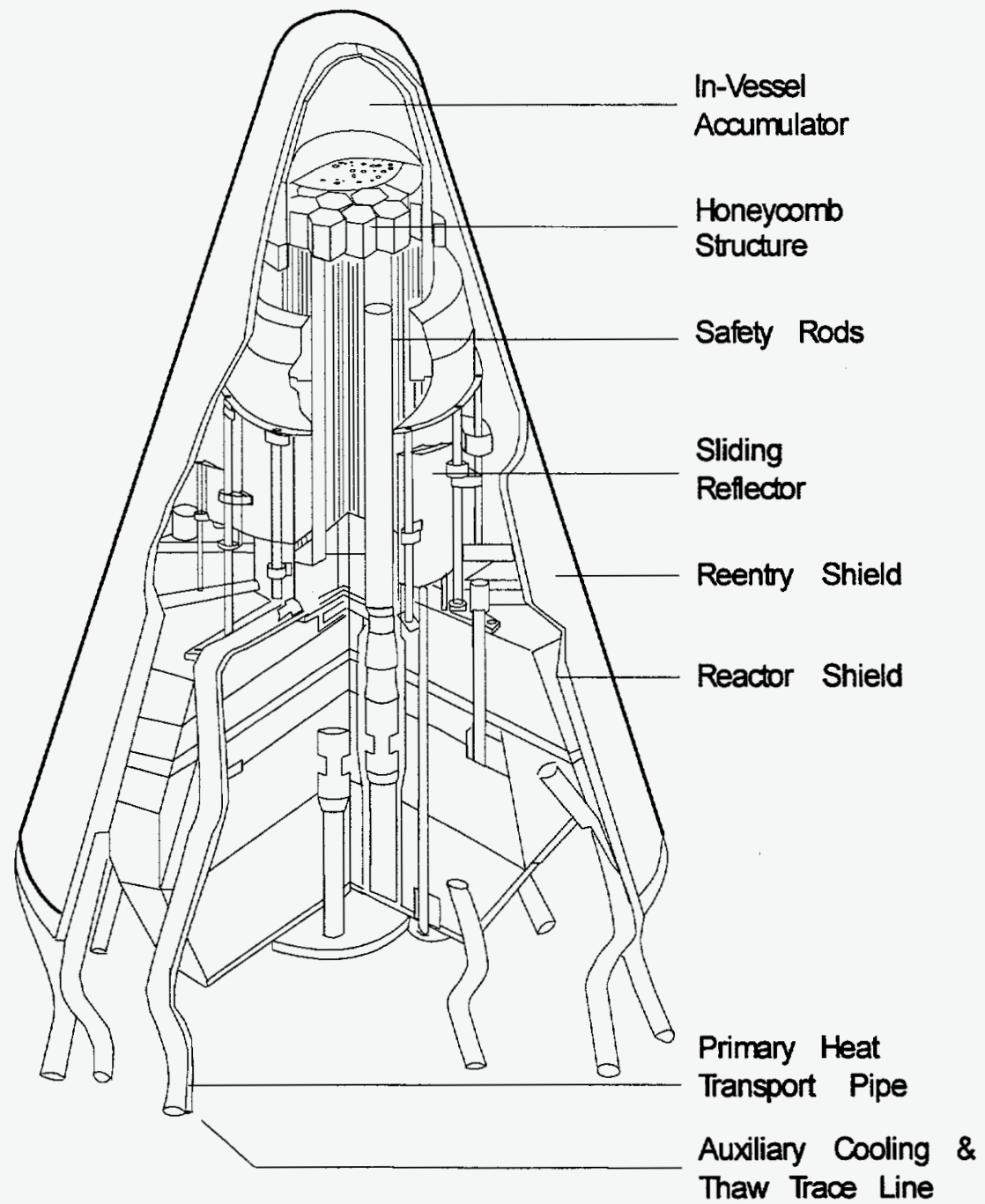

Figure 2. SP-100 Reactor Unit

SP-100 is designed to support a variety of space missions encompassing both national defense and space science objectives. Consequently, the reentry safety policy needed to satisfy a large envelope of missions, ranging from low earth orbit to deep space operations. Both intact reentry and high altitude dispersal were judged to be acceptable design options based on the radiation safety and safeguards risk factors. Calculations demonstrated (Bost et al. 1985), however, that high altitude dispersal was not feasible based on the SP-100 design. While partial break-up leading to a ground footprint was considered acceptable from the safeguards, hazardous materials, and impact criticality criteria, it was not acceptable on the basis of the radiation safety criteria since hot reentry from low earth orbit was not precluded. Intact reentry thus became the chosen criteria and was implemented by shrouding the reactor with a reentry heat shield. Other reentry safety features of SP-100 include reactor startup interlocks to preclude nuclear operations until achieving the required stable orbit, and in-core safety rods that ensure the reactor remained sub-critical if submerged in water or buried in soil. 
A reentry break-up analysis was performed by the General Electric Reentry Systems Group (Temme 1985). This included a six degree of freedom analysis of the reactor system and spacecraft during an orbital decay reentry. Estimates of the break-up altitudes of the boom, radiator, and other reactor system components were performed using proprietary codes. Results of the analytical study indicated that a carbon-carbon reentry heat shield would permit an intact reentry.

\subsection{Summary of Historical Review}

To summarize, reentry safety is provided for by a combination of mechanisms which depend on reactor design and mission requirements. With SNAP-10A and SP-100, reactor start-up was forbidden until achieving a stable orbit. Unlike SNAP-10A, in-core safety rods were employed in SP-100 to maintain a sub-critical reactor configuration during reentry impact and submersion in water or burial in ground. Also unlike SNAP$10 \mathrm{~A}$, a reentry heat shield covers the SP-100 reactor to ensure an intact reentry. The intact reentry mitigates the radiological hazard of a "hot" reentry which is possible with low earth orbits. The NEPSTP reentry safety functional requirements were influenced by the SNAP-10A and SP-100 reentry safety programs. As in SNAP-10A, hot reentry is precluded operationally, which eliminates the necessity of a reentry heat shield, while, as in SP-100, post reentry impact criticality is precluded through incorporation of positive design measures. The following sections will examine cold reentry accidents and discuss why core-configuration constraints are not required for such accidents.

\section{Cold Reentry of TOPAZ II: Breakup Analysis}

A reentry break-up analysis was performed on the TOPAZ II reactor system. This analysis was then used to assess the safety and safeguards risks of cold reentry and to determine if core configuration constraints were warranted for such accidents. Results of the analysis indicate that the TOPAZ II reactor will separate from the balance of the power system and experience partial melting and breakup. However, due to the inherently rugged design of the TOPAZ II thermionic reactor, break-up of the reactor vessel and complete dispersal of the thermionic fuel elements (TFEs) as an aerosol is not predicted. Based on this preliminary analysis, the reentry outcome is bounded on one end by essentially intact reentry and on the other by dispersal of the core but intact reentry of the TFEs, some of which may spill their $\mathrm{UO}_{2}$ pellets. The $\mathrm{UO}_{2}$ pellets are calculated to receive only minor ablation if released from the TFE. This section presents results of the breakup analysis. The following section examines the issue of core configuration constraints.

\subsection{Analysis Assumptions and Methodology}

The orbital decay reentry scenario was chosen for performing the heating and break-up analysis for TOPAZ II. Reentry from orbital decay could occur due to several causes. The principal cause would be due to failure of the launch vehicle to deploy the satellite into its proper orbit. A low altitude orbit or an elliptical orbit with a low altitude perigee 
will decay over a period of days or weeks. Of course, failure of the launcher at any point along its trajectory will bring TOPAZ II falling back to earth, but the orbital decay scenario produces higher reentry velocity than typical launcher aborts. Since aerodynamic heating scales as the velocity cubed, the orbital decay scenario will also generate greater heat loads on TOPAZ II.

A thrust misalignment malfunction of the launcher's upper stage during orbital insertion could cause a steep angle reentry. This would lead to higher peak heating rates and dynamic pressures than orbital decay, while orbital decay results in a larger overall time-integrated heat input. Orbital decay yields a more severe environment from the perspective of melting while steep angle reentry would expose the system to a more severe aeromechanical environment. Orbital decay was chosen for this preliminary analysis because it is considered to be the "worst case" scenario from the standpoint of causing break-up and dispersal of TOPAZ II. Steep angle reentry scenarios will be considered in the next phase of the safety analysis program. Analysis will also be performed on the credibility of reentry accidents due to collision with space debris and due to misuse of the electric thrusters.

\subsection{Aerodynamic Assumptions}

Several assumptions were made that simplify the analysis yet do not substantially degrade the validity of the results. They will be explained with the assistance of Figures 3 and 4 which depict the TOPAZ II power system and reactor unit.

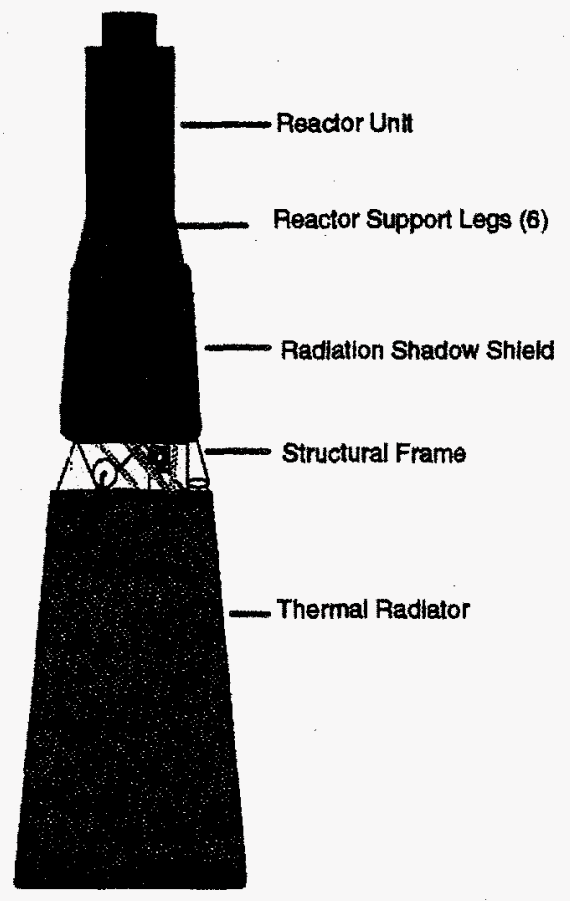

Figure 3. TOPAZ II System

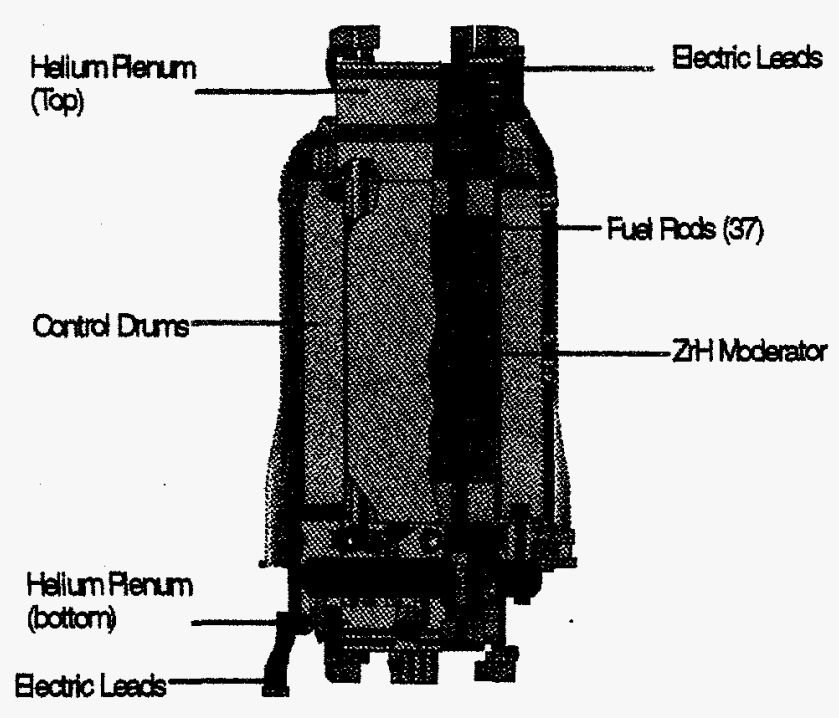

Figure 4. TOPAZ II Reactor Unit 
The power system (i.e. reactor unit, radiation shield, thermal radiator, and structure) is assumed to separate from the spacecraft at high altitude. Consequently, the balance of the spacecraft is not included in the breakup analysis. The radial reflector/control drum assembly is held in place by two thin stainless steel binding tapes, very similar to the SNAP-10A design. Reentry tests and analysis performed on SNAP-10A showed that the tapes failed and released the reflector assembly at approximately $100 \mathrm{~km}$, the threshold altitude for aerodynamic heating. Therefore, in the TOPAZ II reentry analysis, the reflectors were assumed to be released at $100 \mathrm{~km}$ and were not incorporated into the subsequent aerothermal analysis of the reactor vessel.

The TOPAZ II power system was assumed to fly a stable, reactor first, zero angle of attack trajectory. Analysis of systems with similar geometric and mass properties justifies this assumption. The stable, reactor first trajectory exposes the reactor vessel to higher heating loads, yielding a worst case scenario from the standpoint of reactor vessel breakup. The reactor unit was assumed to perform a Random-Tumble-and-Spin (RTS) reentry if and when it separated from the system. The length-to-diameter ratio of the reactor is such that stable flight, either end-on or side-on is unlikely. Figure 5 presents an overview of the breakup assumptions.

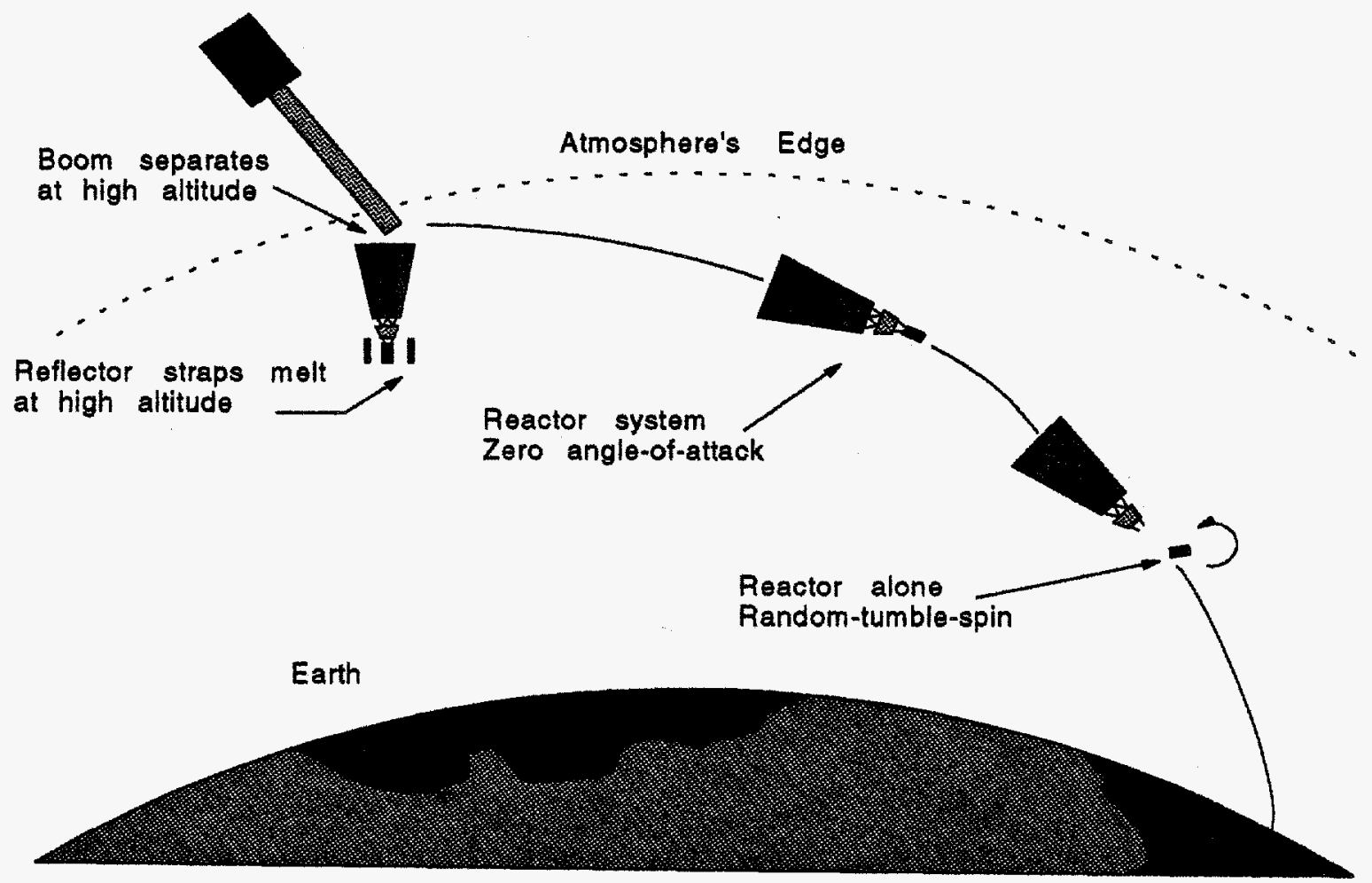

Figure 5. Reentry Breakup Assumptions 


\subsection{Heating/Thermal Response Assumptions}

As depicted in Figure 3, the reactor unit is attached to the top of the radiation shadow shield by six support legs. The legs are a titanium alloy $0.5 \mathrm{~cm}$ in thickness, and bolt to stainless steel flanges which are an integral part of the shadow shield. The reactor unit is assumed to separate from the system when the legs reach their melt temperature. The legs are canted out at an angle of approximately 16 degrees relative to the vertical axis of the system. The region between the reactor and radiation shield is aerodynamically modeled as a 16 degree half angle frustum and the heating to this region is computed based on the flow field about the frustum.

Referring now to Figure 4, the entire reactor vessel is composed of stainless steel with the top of the vessel consisting of three layers, each with a nominal thickness of 1.5 $\mathrm{mm}$. The top of the reactor vessel is assumed to be compromised when all three layers have reached the melt temperature. The region between the top and intermediate layer is called the top helium plenum and is packed tightly with electrical connectors made of copper. This zone was modeled as being filled with copper at 70 percent nominal density. The region between the intermediate layer and bottom layer forms the NaK upper plenum. The NaK was assumed to have leaked out of the plenum due to failure in the external NaK piping loop.

The bottom of the reactor vessel is formed by four layers of stainless steel with the same nominal thickness as used for the top of the vessel. The first two layers comprise the cesium gas plenum while the second two layers make up the NaK bottom plenum. The region between the two plenums is called the bottom helium plenum and contains the copper electrical connectors similar to the top helium plenum and was modeled in the same manner. Structural integrity of the bottom section is lost when all four layers of stainless steel have reached the melt temperature. The top and bottom reactor sections are fastened together via the NaK internal piping, which consists of 37 annular flow paths formed by 37 sets of concentric stainless steel tubes each of which is welded to the top and bottom NaK plenums. The NaK internal piping provides a design which is structurally more robust than US. space reactors. Melt-through of the sidewall of the TOPAZ II reactor will have little effect on the break-up since the NaK internal piping provides the bulk of the structural strength.

TOPAZ II reactor break-up is assumed to occur when both top and bottom of the reactor vessel have melted. This involves melting through three plates of $1.5 \mathrm{~mm}$ stainless steel for the top of the reactor and four plates on the bottom. When the plates comprising the NaK plenums reach melt, the welds that attach the internal NaK piping are also assumed to fail. The combined effects associated with the aerodynamic flow forces and the tumbling of the reactor are assumed to disperse the core. This predicts a lower than actual failure altitude since the aeromechanical forces could disassemble the reactor before complete melting occurs. However, the error in predicting failure altitudes caused by this assumption are within the bounds of this first order analysis. 


\subsection{Methodology}

The analysis methodology can be divided into three sections: trajectory analysis, heating analysis, and thermal response analysis. To determine the trajectory of the initially intact reactor system, the initial reentry state vector must be known along with the system's aerodynamic coefficients. These trajectory data are input to a boundary layer flow field code which computes the time-dependent heating rates to selected locations along the surface of the reactor system. The heating data are then input to a thermal response code which performs the thermal analysis and computes the timedependent temperature profile at selected locations on the system. When the reactor separates from the balance of the system, a new trajectory must be calculated based on the ballistic characteristics of the reactor alone and the state vector of the reactor at the separation point. Breakup analysis of the reactor then follows the same steps followed by the overall system.

\subsection{Results}

Figure 6 presents the thermal response of the reactor support legs, showing that failure begins at approximately 672 seconds. During this time period, the top of the reactor vessel is exposed to stagnation point reentry heating.

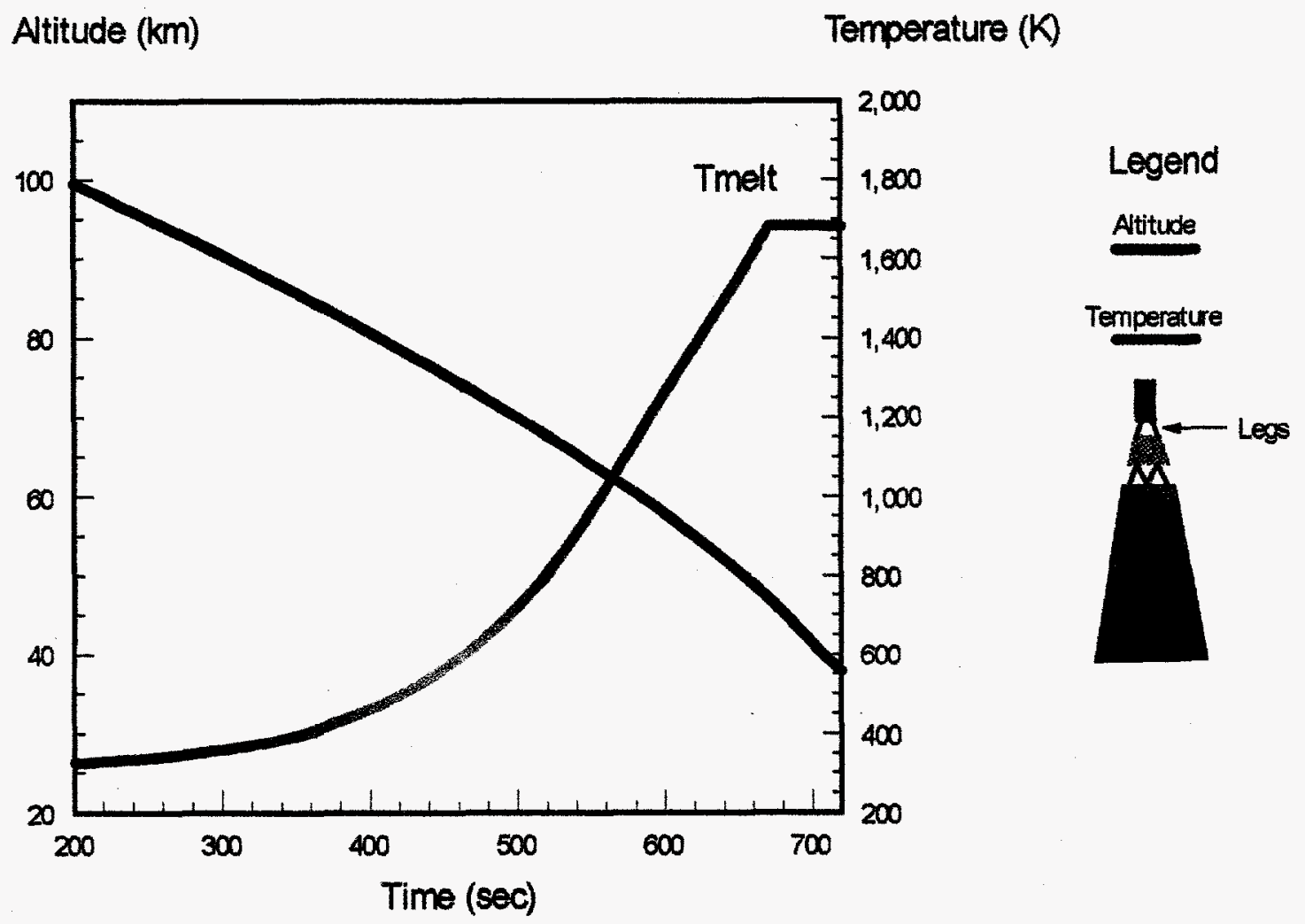

Figure 6 . Thermal Response of Reactor Support Legs 
Thermal response calculations for the top of the vessel are shown in Figure 7 and indicate that complete melt-through of all three stainless steel plates occurs at approximately 662 seconds, just 10 seconds before failure of the support legs. Therefore, the top of the vessel is completely destroyed prior to the release of the reactor unit from its support legs.

Altitude (km)

Temperature $(\mathrm{K})$

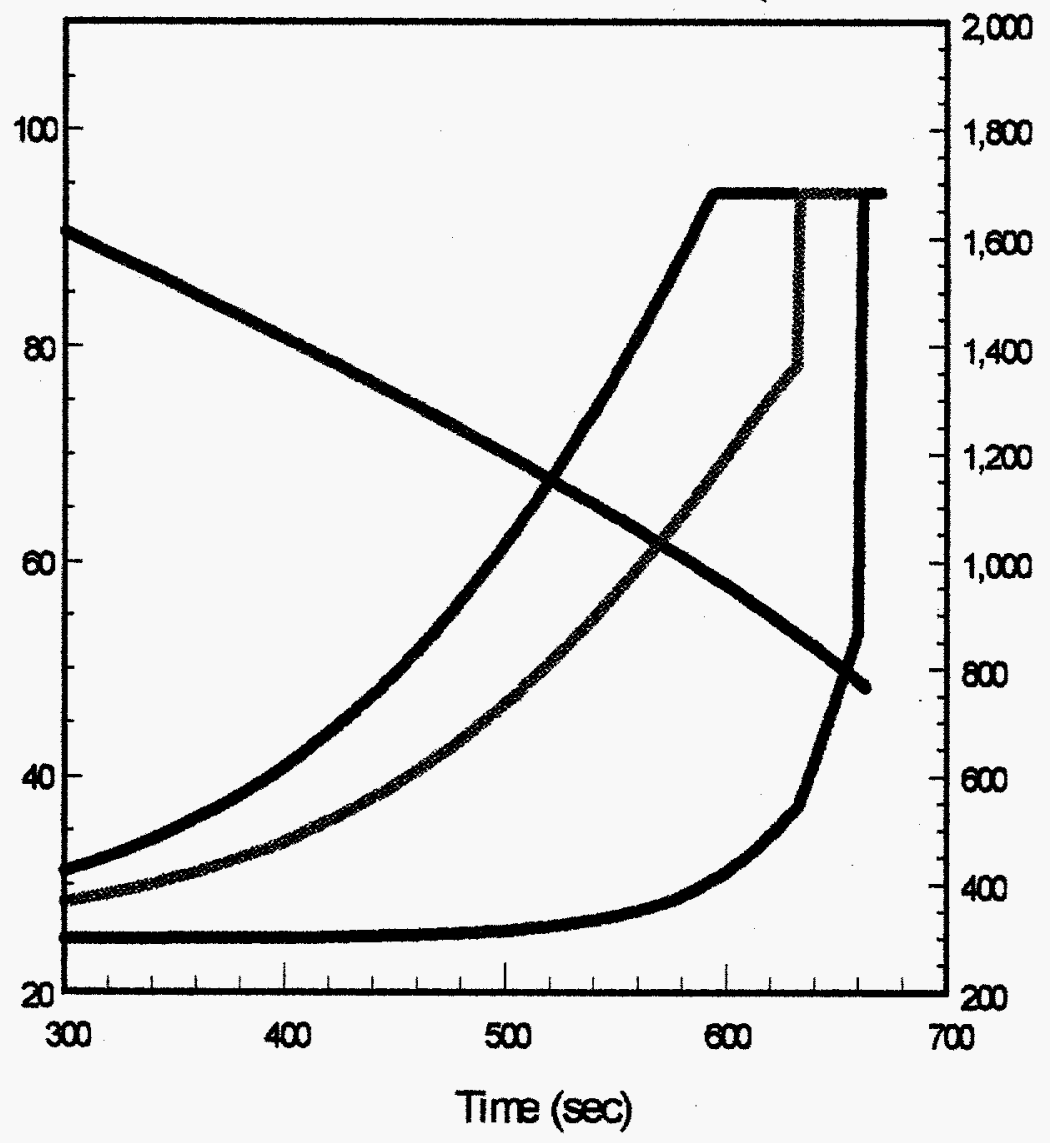

Legend

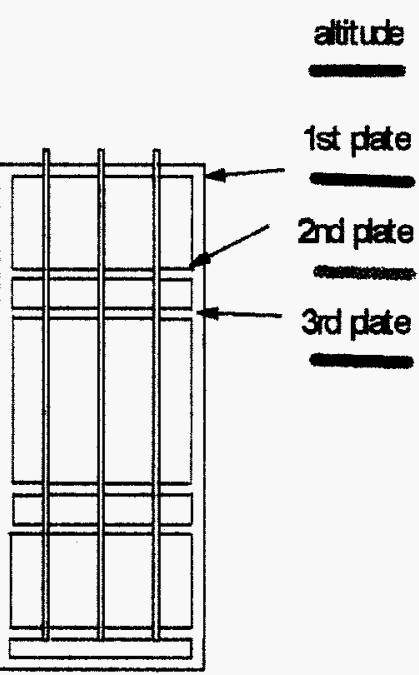

Figure 7. Thermal Response of Reactor Vessel Top Section

The reactor begins its RTS trajectory at 672 seconds. The bottom of the reactor as well as the sidewalls are still intact while the top section has melted through to the top of the axial beryllium reflector. It is possible that the reflector, moderator blocks, and some fuel pellets could slide out from the open top of the reactor during RTS motion. It is also possible that differential thermal expansion due to reentry heating will prevent the core from dispersing or that core melt will resolidify and seal the top of the TFEs preventing release of the $\mathrm{UO}_{2}$ pellets. Resolving issues at this level of detail will require more sophisticated modeling tools and probably wind tunnel/arc heater testing. For this preliminary analysis the assumption, as stated above, was that dispersal of the core 
does not occur until both the top and bottom of the vessel have been compromised. Thermal response analyses on the reactor sidewall and bottom section during the RTS phase indicate that only a portion of the structure reaches melt temperature before the lower ballistic coefficient of the reactor (compared to that of the whole system) reduces its speed below the level that supports aerothermodynamic breakup. Figure 8 presents the thermal response of the vessel sidewall during the RTS phase and shows that the outer surface of the vessel reaches melt temperature at approximately 705 seconds while the inner surface in contact with the $\mathrm{ZrH}$ remains below the melting point.
Altitude (km)
Temperature (K)

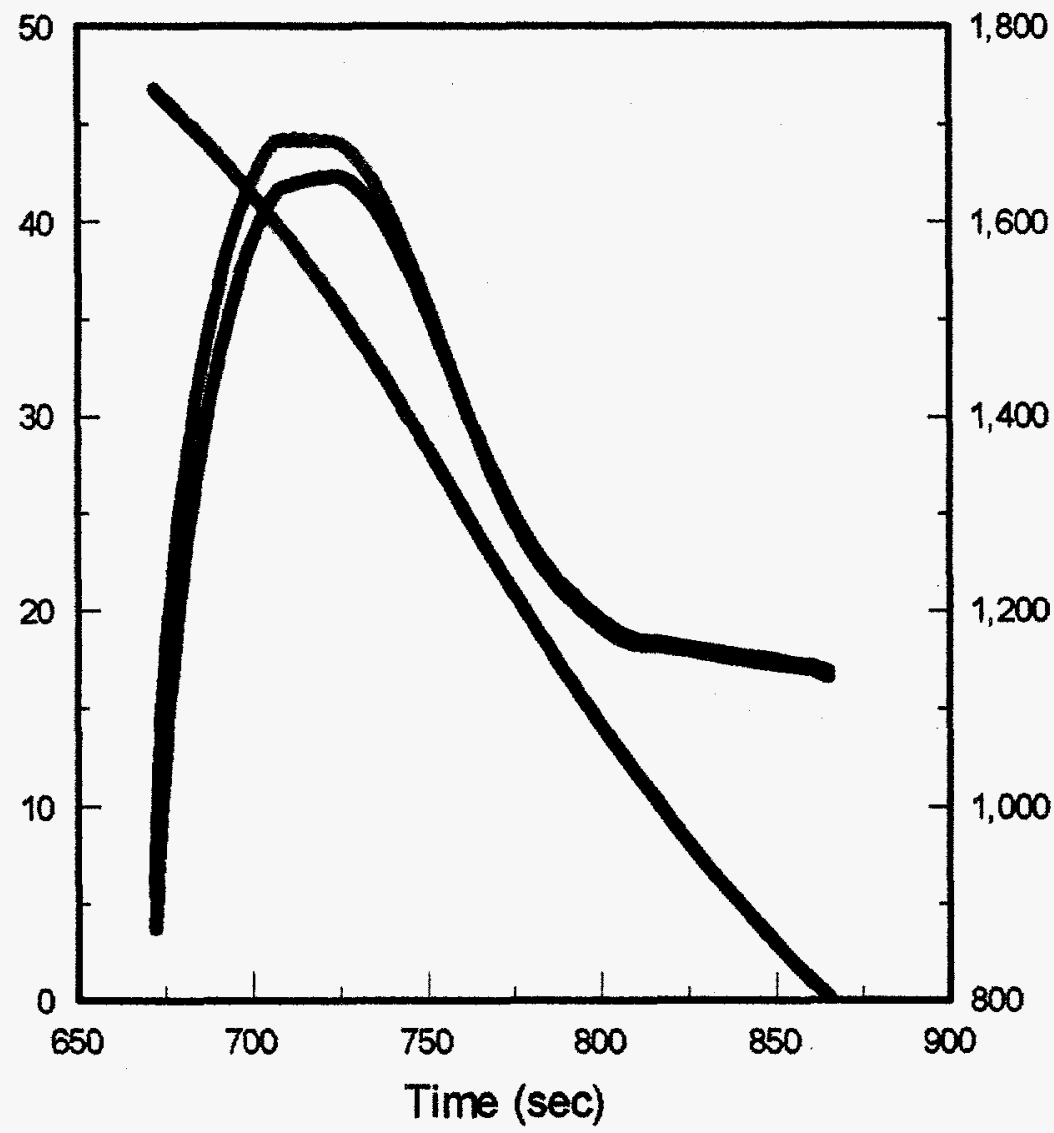

Legend

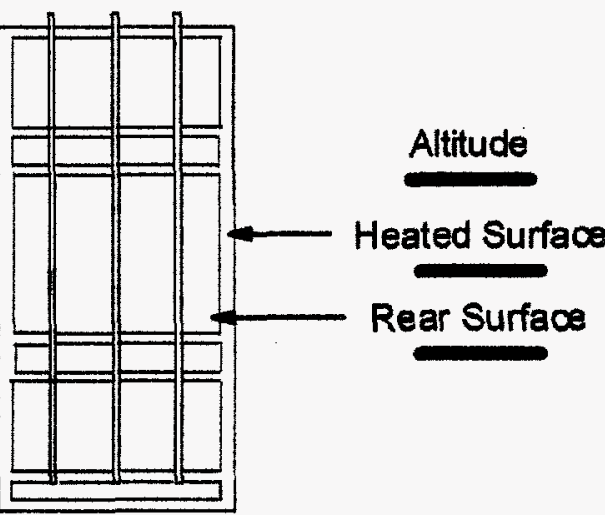

Figure 8. Thermal Response of Reactor Vessel Sidewall 
As depicted in Figure 9, the first plate on the bottom of the reactor melts-through at approximately 725 seconds, but the remaining three layers experience no melting. Figure 10 depicts the overall break-up history superimposed on a plot of trajectory.

Altitude $(\mathrm{km}) \quad$ Temperature $(\mathrm{K})$

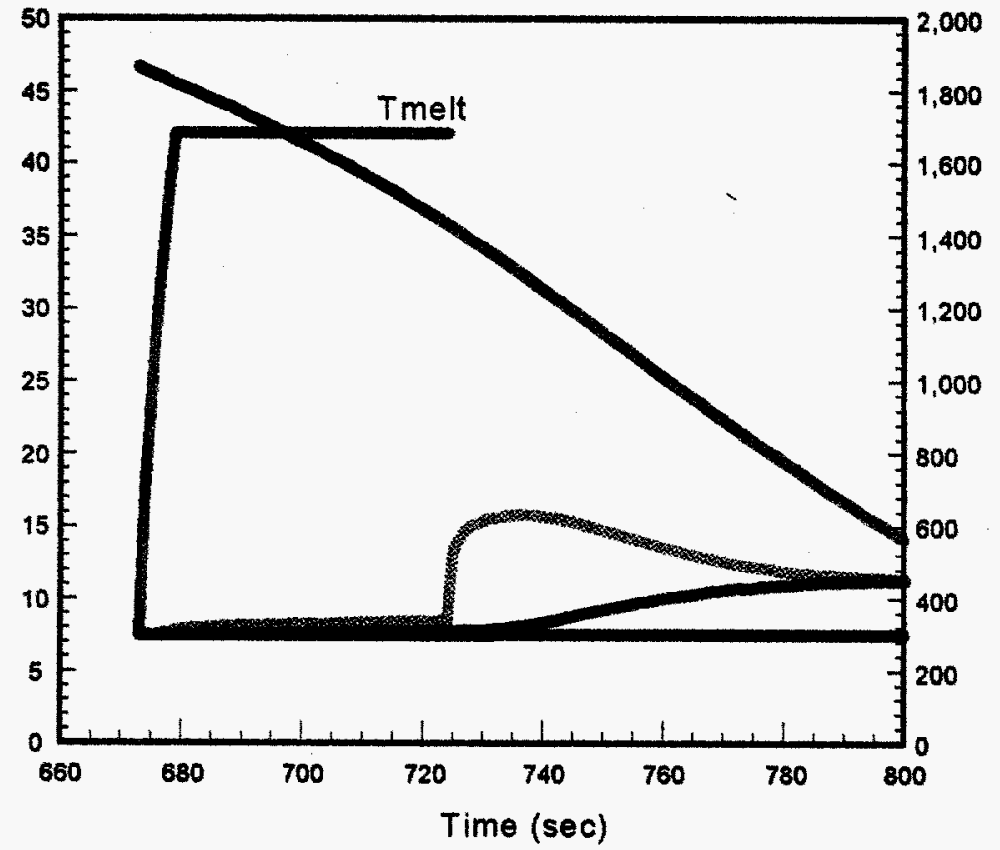

Legend

Figure 9. Thermal Response of Reactor Vessel Bottom Section

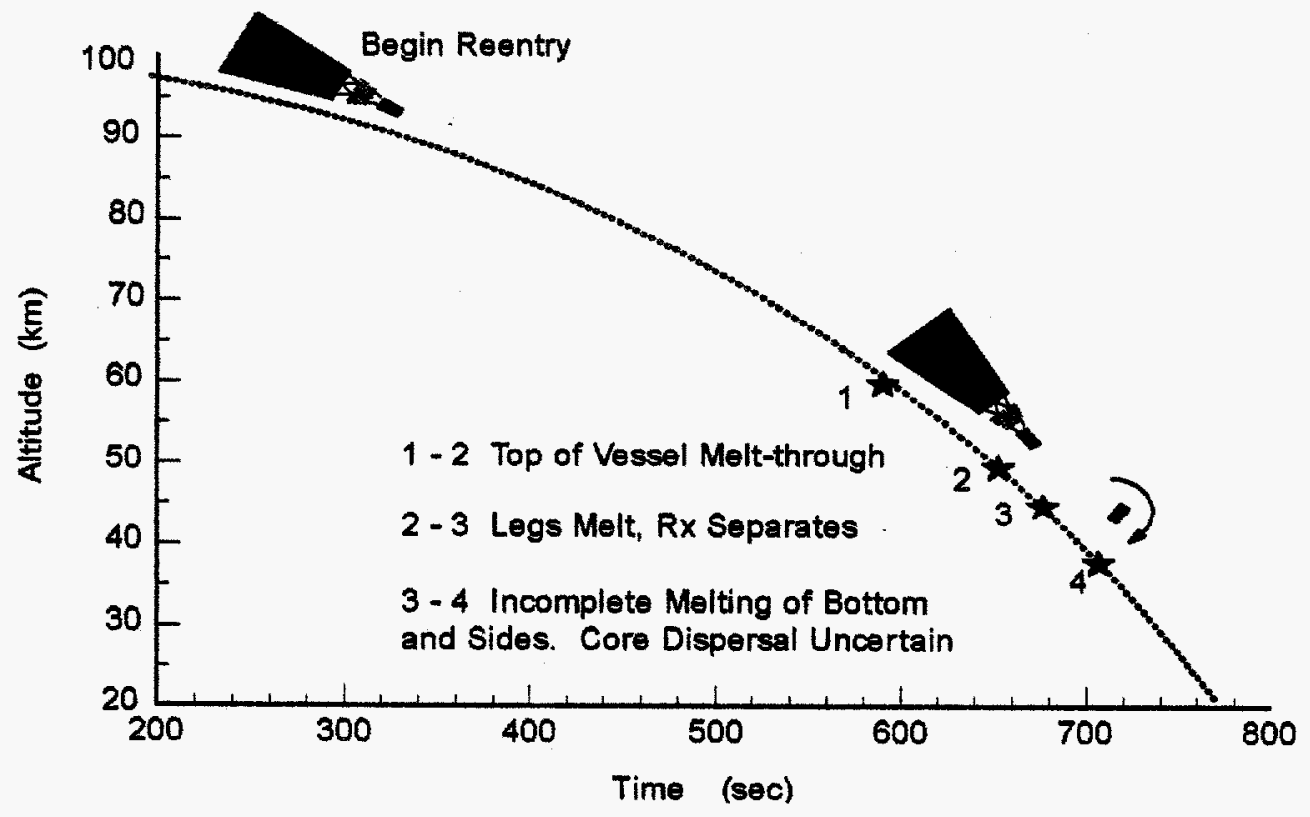

Figure 10. TOPAZ II Breakup Sequence 


\subsection{Ablation of $\mathrm{UO}_{2}$ Particles}

Heating and thermal response analyses were also carried out on the $\mathrm{UO}_{2}$ pellets in order to determine the percent mass loss as a function of core release altitude. Dispersal and ablation of the fuel into aerosol-sized particles would lower the safeguard risks in addition to removing the problem of impact criticality. A computer code was used (Connell, 1984) which couples the trajectory to the thermal analysis by recomputing the ballistic coefficient as mass is ablated from the fuel pellets. The initial conditions for each calculation become the altitude of release along with the velocity and flight path angle of the reactor at this point. Figure 11 presents a summary of the results of a series of code runs and depicts the percent ablation as a function of the release altitude. The results show that significant (i.e. exceeding $10 \%$ ) $\mathrm{UO}_{2}$ pellet ablation will only occur in a narrow altitude corridor between 52 and $63 \mathrm{~km}$. Below this altitude, the release velocity is too low to generate significant heating. Above this band, air density is too low to support significant heating, but is still sufficient to decelerate the pellet (whose ballistic coefficient is very small) such that when the particle reaches the dense layers of atmosphere, the pellet velocity is insufficient to support melting and ablation.

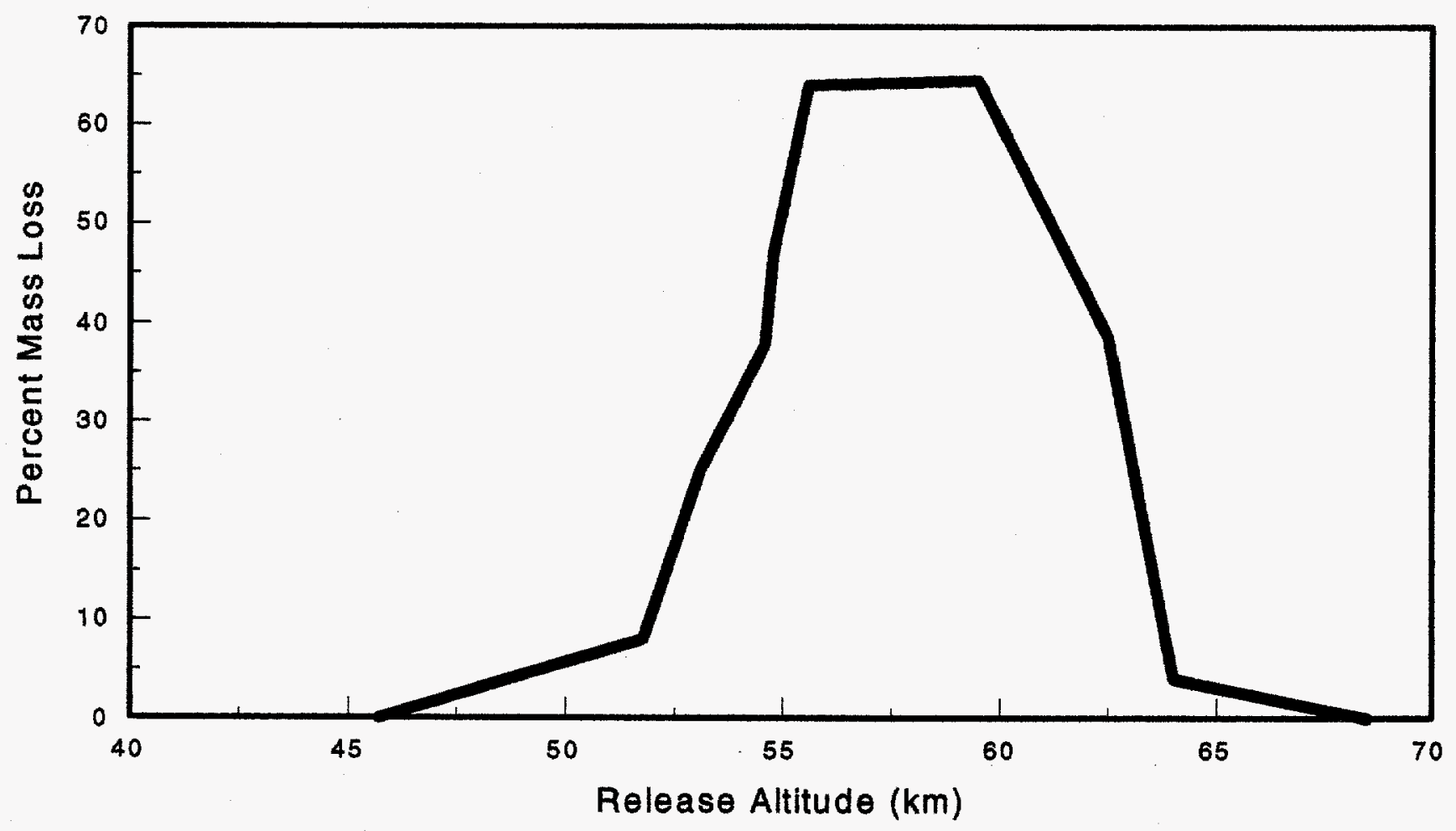

Figure 11. $\mathrm{UO}_{2}$ Pellet Ablation Parametric Analysis 


\subsection{TOPAZ II Reentry Breakup Summary}

The calculations performed indicate that the reactor experiences partial melting and ablation from an orbital decay reentry. The final fate of the reactor is difficult to discern without testing and further analysis. In its weakened state, aerodynamic pressure and flow forces, in conjunction with the centrifugal forces created by RTS, may be sufficient to disassemble the core. On the other hand, there may be sufficient residual strength to hold the core together until ground impact. It is clear, however, that complete dispersal and disintegration of the TOPAZ II core is not a possibility without active design features, such as explosive interaction. The fate of the reactor core lies somewhere between the two bounding states of essentially intact and what will be termed "ground dispersal." Ground dispersal is defined as the condition in which the core materials are released from the reactor vessel but survive reentry leaving a footprint of debris dispersed on the earth's surface. The amount of ablation mass loss arising from the reentry of the individual TFEs was not computed, but due to the refractory materials used in the TFEs, and the low altitude at which they would be released, the percent mass loss would be small. The same conclusion applies to the $\mathrm{UO}_{2}$ pellets. Figure 11 showed that only minute ablation would occur for pellet release altitudes below $50 \mathrm{~km}$. Since the earliest possible release of fuel pellets is at $50 \mathrm{~km}$, the altitude of failure for the top of the reactor, almost no ablation is expected.

As stated in the section on assumptions, aeromechanical forces were not computed and their contribution to bringing about the break-up of TOPAZ II was not coupled into the analysis in any quantitative manner. Analysis tools are available to perform such coupled analyses but were beyond the scope of this preliminary effort. Of course, the assumption that core dispersal occurs when top and bottom of the reactor vessel have reached melt temperature relies implicitly on the aerodynamic forces to remove the core materials from the remaining weakened structure, whose strength may still be considerable.

Many simplifying assumptions were made, most of which tended to over-predict the heating and increase the likelihood of reactor core release. The assumption that aeromechanical effects are small compared to aerothermal effects is the only assumption that does not result in enhancing core dispersal. This, however, is a fairly standard assumption for first order reentry break-up analysis and inclusion of the aeromechanical effects would not significantly alter the breakup results or the conclusions to be drawn from them.

\section{Analysis of the Need for Cold Reentry Core Configuration Constraints}

The results from the above analysis were used to determine the importance of reactor core configuration constraints for cold reentry accidents. Two core constraint options were postulated, essentially intact and complete dispersal at high altitude. Intact reentry is obtained by shrouding the reactor vessel with an ablative heat shield. Complete dispersal of the core at high altitude would require more intrusive methods, 
especially when attempting to retrofit onto an existing system. For TOPAZ II, the only core constraint option given serious consideration was intact reentry through the application of a heat shield. The decision not to require core constraints for cold reentry was based on comparing the relative risks of intact vs. nonconstrained reentry. The breakup analysis showed that an unconstrained core will result in a ground dispersal of the reactor core material and possibly some atmospheric release of the virgin HEU. A core-constrained reentry will not release HEU to the atmosphere, but faces the risk of a criticality accident. The next section will examine the relative risk factors. Radiological safety issues will be addressed first, followed by the safeguards issue.

\subsection{Cold Reentry Radiological Safety Risks}

\section{Impact Criticality Safety}

As stated in the introduction, two radiation safety risk factors were identified for cold reentry accidents: impact criticality safety and atmospheric dispersal/aerosolization of the HEU which poses an inhalation toxicology risk. From the perspective of impact criticality, any disassembly of the core material would reduce the chances of obtaining a critical geometry upon impact. However, provided the anticriticality device functions properly, impact criticality risks would also be low for an intact reentry. A qualitative examination indicates that the relative risks are about equal. If the core configuration is unconstrained during reentry accidents, and reentry heating causes a breach of the reactor vessel leading to a partial or full dispersal of the core, the risk of a criticality excursion upon ground or water impact is very small. If the core reenters intact, either because of a core constraint system or because of insufficient reentry heating on an unconstrained core, then the anti-criticality mechanism will prevent a criticality accident.

\section{Atmospheric Dispersal of Virgin HEU: Inhalation Toxicology Safety}

Irrespective of the radiological hazards, uranium is a heavy metal poison which attacks the kidney, leading to acute renal damage and fatality at high exposure levels. At less than 10 percent $U-235$ enrichment, the heavy metal hazard governs the exposure level limits. Above 10 percent, the exposure limits are based on radiological hazards which become more restrictive than the heavy metal limits. Since TOPAZ II fuel is 96 percent enriched, the radiological inhalation hazards govern.

Focusing, therefore, on the radiological aspects, uranium emits an alpha particle with an energy $(4.5 \mathrm{MeV})$ that is fairly independent of the uranium isotope. The alpha activity, however, is dependent on the isotopic composition of the uranium metal. Figure 12 demonstrates that the specific activity of uranium increases with U-235 enrichment (Marshall, 1992). Table I explains this phenomena by showing the isotopic composition and specific activity of natural uranium (number in parentheses) in comparison to TOPAZ II HEU. 


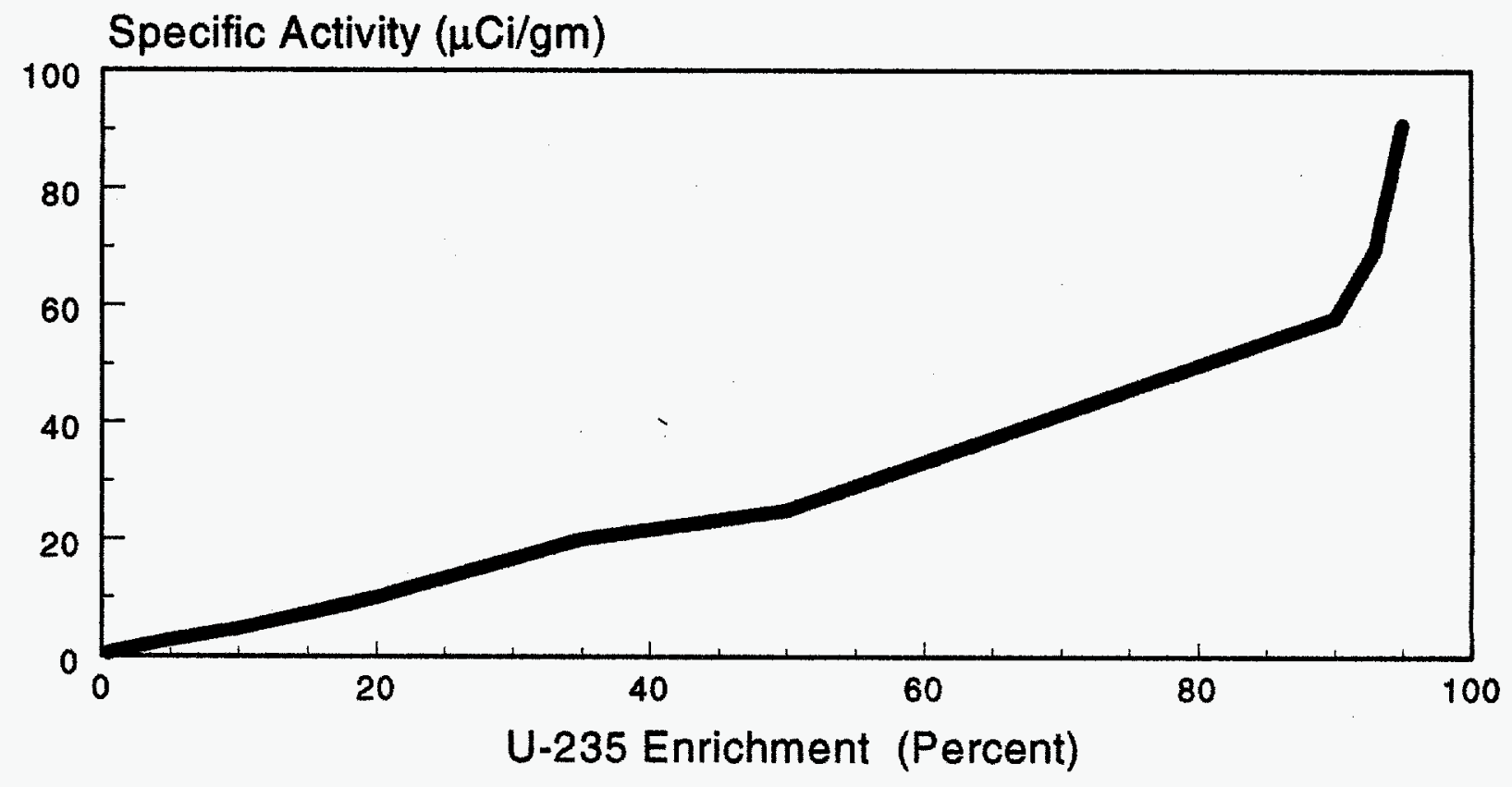

Figure 12. Specific Activity of Enriched Uranium

Table I. Specific Activity of Virgin Reactor Fuel vs. Natural Uranium

\begin{tabular}{|c|l|c|c|}
\hline Isotope & Mass \% & $\begin{array}{c}\text { T1/2 } \\
\text { Years }\end{array}$ & $\begin{array}{c}\text { Specific Activitv } \\
\mu \mathrm{Cl} / \mathrm{g}\end{array}$ \\
\hline$U$ U-234 & $1.6(0.0054)$ & $2.44 \mathrm{EE}$ & $98.8(0.33)$ \\
\hline$U-235$ & $96(0.72)$ & $7.04 \mathrm{E} 8$ & $2.08(0.016)$ \\
\hline$U-238$ & $2.1(99.3)$ & $4.47 \mathrm{E} 9$ & $0.01(0.33)$ \\
\hline \multicolumn{4}{|c|}{ Total $101(0.68)$}
\end{tabular}


This shows that the main cause of the increased activity is the collateral enrichment of U-234 which accounts for 98 percent of the activity, resulting in a two order increase relative to natural uranium. Using $100 \mu \mathrm{Ci} / \mathrm{gm}$ as a "ballpark" number for the activity of the TOPAZ II HEU, the entire core, containing approximately $26 \mathrm{~kg} \mathrm{HEU}$, has an intrinsic activity of $2.6 \mathrm{Ci}$. To put this in perspective, an RTG of the type used to power the Galileo and Ulysses satellites uses Pu-238 as the alpha emitting heat source which has an inherent specific activity of $14 \mathrm{Ci} / \mathrm{gm}$. For a typical Pu-238 load out of $10 \mathrm{~kg}$ (about $4.5 \mathrm{kWth}$ ) the RTG contains a total activity of $1.4 \mathrm{E} 5 \mathrm{Ci}$. Therefore, the intrinsic activity of TOPAZ II is quite benign in comparison with RTG space nuclear power sources which have flown safely for decades.

Alpha particles in the $5 \mathrm{MeV}$ energy range have a very short stopping distance, about 3 $\mathrm{cm}$ in air or $30 \mu \mathrm{m}$ in tissue, and can easily be stopped within the outer layers of skin. Therefore, $\mathrm{UO}_{2}$ poses only minor health risks so long as it remains external to the body. Even if ingested, the mucous layers which coat the stomach and intestinal walls are of sufficient thickness to shield internal organs; and since $\mathrm{UO}_{2}$ is highly insoluble, even in stomach fluid, it will pass through the body without being absorbed. Therefore, the dominant health risk associated with exposure to $\mathrm{HEU}$ in oxide form is through inhalation of respirable particles which could form during reentry. Figure 13 diagrams the inhalation pathways of $\mathrm{UO}_{2}$ aerosol as a function of particle size (Langham, 1967), showing that only particles of diameter $2 \mu \mathrm{m}$ and less reach the lower lung and are retained in the body. Some of these particles will be taken up by the blood stream and translocated to other body organs. Over the course of a few years the retained $\mathrm{UO}_{2}$ will be deposited, in roughly equal proportions, in the lung, lymph nodes, liver, and bone.

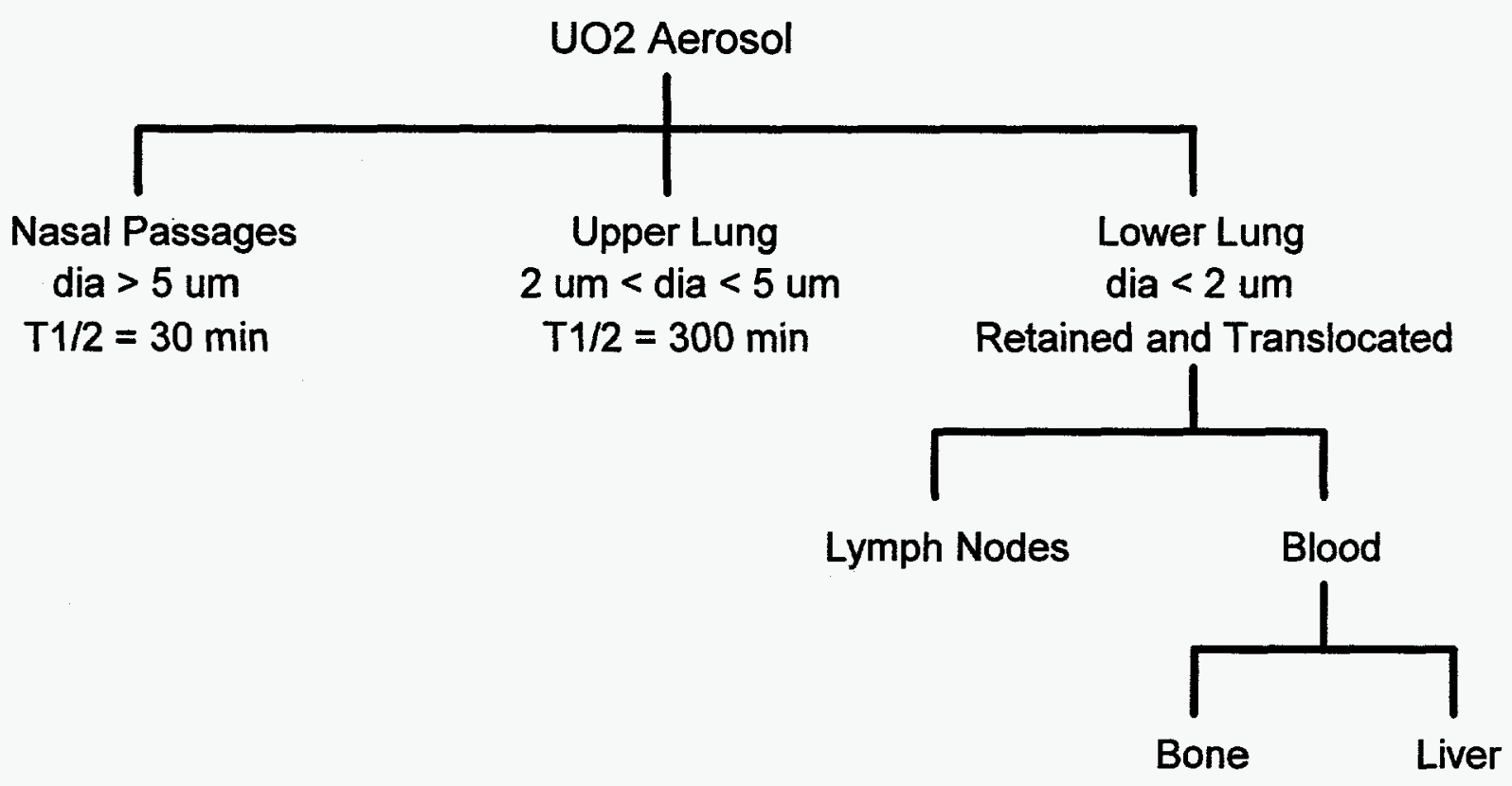

Figure 13. $\mathrm{UO}_{2}$ Aerosol Inhalation Pathways 
The lung and bone are the most sensitive organs for alpha radiation damage. The criterion used to formulate the DOE Derived Air Concentration (DAC) limits for uranium particulates is the prevention of damage to these organs. Alpha radiation damage to the lung results in an increased risk of cancer fatality. This is termed a stochastic effect which is governed by a probability density function. Theoretically, stochastic effects such as cancer fatality exhibit no threshold dose limit and no dose-effect relationship-an increase in dose will translate to an increased risk of cancer fatality but not an increase in the amount or severity of the cancer. Conversely, nonstochastic effects do exhibit a threshold dose level, below which the effect is not observed, and a dose-effect relationship. $\mathrm{UO}_{2}$ particles translocated to the bone produce nonstochastic damage to the bone surface in the form of surface lesions and tumors with a bone dose threshold of $50 \mathrm{rem}$.

The DOE DAC limits are given in units of $\mu \mathrm{Ci}$ per $\mathrm{ml}$ of air and are a function of the radiation source material and its solubility in the lung. Table II (Eckerman, 1988) compares the DAC limits for uranium and plutonium. The solubility class $(D=$ days, $W=$ weeks, $Y=$ years) is defined in terms of the half-time, $T 1 / 2$, of the particular metal compound within the lung, defined as the time required for the mass of material to decline to half of its initial value. The solubility of the metal compound, along with the relative biological effectiveness of the radioisotope on the given organs, governs the establishment of the DAC limit. In the case of plutonium, which is approximately ten times more effective than uranium at producing nonstochastic bone damage, a higher solubility means a quicker translocation to the bone, greater bone exposure, and therefore a lower DAC limit. For uranium, stochastic effects on the lung govern the DAC limit except for class $D$, the most soluble class, for which nonstochastic bone effects dominate. The DAC limit for uranium is therefore observed to increase with increasing solubility.

Table II. DOE Derived Air Concentration Limits for Plutonium and Uranium

\begin{tabular}{|l|l|l|l|}
\hline & $\begin{array}{l}\text { Class D Solubility } \\
\text { Clearance T1/2 Days }\end{array}$ & $\begin{array}{l}\text { Class W Solubility } \\
\text { Clearance T1/2 Weeks }\end{array}$ & $\begin{array}{l}\text { Class Y Solubility } \\
\text { Clearance T1/2 Years }\end{array}$ \\
\hline $\mathrm{PU}$ & & $\begin{array}{l}2 \mathrm{E}-12 \mathrm{uCi} / \mathrm{ml} \\
\text { Nonstochastic, Bone }\end{array}$ & $\begin{array}{l}\text { 6E-12 uCi/ml } \\
\text { Nonstochastic, Bone }\end{array}$ \\
\hline $\mathrm{U}$ & $\begin{array}{l}5 \mathrm{E}-10 \mathrm{uCi} / \mathrm{ml} \\
\text { Nonstochastic, Bone }\end{array}$ & $\begin{array}{l}\text { 3E-10 uCi/ml } \\
\text { Stochastic, Lung }\end{array}$ & $\begin{array}{l}\text { 2E-11 uCi/ml } \\
\text { Stochastic, Lung }\end{array}$ \\
\hline
\end{tabular}


For the purpose of bounding the uranium inhalation health risks, it is assumed that the entire core is released and aerosolized into respirable-sized particles. Using the most limiting DOE DAC of $2 \mathrm{E}-11 \mathrm{uCi}$ per ml-air and the total inherent activity of $2.6 \mathrm{Ci}$, the volume of air required to disperse and dilute the aerosol below the DAC is:

$$
2.6 E 6 \mu C i \times \frac{m l}{2 E-11 \mu C i} \times \frac{1 \mathrm{~cm}^{3}}{1 \mathrm{ml}} \times \frac{1 \mathrm{~km}^{3}}{1 E 15 \mathrm{~cm}^{3}}=130 \mathrm{~km}^{3}
$$

Therefore, as an upper bound, the respirable aerosol formed during the reentry must be dispersed over a volume of air space roughly $5 \mathrm{~km}$ on a side. Since aerothermodynamic considerations limit the altitude of aerosol formation to the $60 \mathrm{~km}$ regime (see Figure 11), any $\mathrm{UO}_{2}$ aerosol formed will most certainly be dispersed over an air volume far in excess of the $130 \mathrm{~km}^{3}$ requirement. The health risk of $\mathrm{UO}_{2}$ inhalation from a cold reentry accident of an unconstrained core is therefore negligible, both from an absolute perspective and relative to a core-constrained cold reentry.

To summarize, two radiological safety risk factors were examined: impact criticality and $\mathrm{UO}_{2}$ aerosol inhalation. In both cases the relative risk comparison of cold reentry accidents with and without core constraints showed no significant differences. Regardless of whether the core remained intact or dispersed upon reentry, the risks were about the same and were found to be negligible. For cold reentry accidents, no safety advantages were obtained by constraining core configuration.

\subsection{Relative Safeguards Risks}

An important part of the safeguards analysis for space nuclear power sources is an examination of the effects of satellite reentry. When Cosmos 954 reentered, it scattered radioactive material along a path over $1000 \mathrm{~km}$ long. A space reactor using highly enriched uranium as fuel could scatter weapons-grade Special Nuclear Material (SNM) as it reentered, making recovery of the material by a potential proliferant state possible.

Figure 14 shows a Monte Carlo analysis of the probability of SNM diversion performed by Los Alamos (Sullivan, 1985) for the SP-100 program. For this analysis, 100,000 histories were run with the reentry impact point and swath length selected randomly from a parameter space constrained by the pre-reentry orbital elements and by reentry physics. Two initial load outs of SNM, $100 \mathrm{~kg}$ and $200 \mathrm{~kg}$, were chosen as representative of SP-100 designs, and were assumed to be uniformly distributed along the swath. The International Atomic Energy Agency (IAEA) standard of $25 \mathrm{~kg}$ was chosen to represent a significant quantity for diversion and weaponization. 
This analysis indicates that the probability of diversion is fairly constant for footprints less than $500 \mathrm{~km}$ (roughly 300 miles) but rises sharply reaching a maximum at approximately $1800 \mathrm{~km}$ (1100 miles). The peak value is dependent on and scales roughly with the initial SNM load out. Since the total SNM load out for TOPAZ II of 26 $\mathrm{kg}$ is just slightly above the IAEA standard, one would not expect its diversion probability curve to exhibit this peaking phenomenon. Instead, it should start at 5 percent and gradually roll-off as the footprint length increases, thus preventing $25 \mathrm{~kg}$ from falling within the borders of a proliferant state. If, however, a more stringent definition of a significant quantity is used--5 kg or even $2.5 \mathrm{~kg}$--then the TOPAZ II diversion probability curve should behave similarly to those shown in Figure 14 . Indeed, assuming rough linear scaling, the $100 \mathrm{~kg}$ reactor curve would represent TOPAZ II for a significant quantity definition of approximately $6.5 \mathrm{~kg}$, and the upper curve would represent TOPAZ II if a significant quantity were defined to be $3.25 \mathrm{~kg}$.

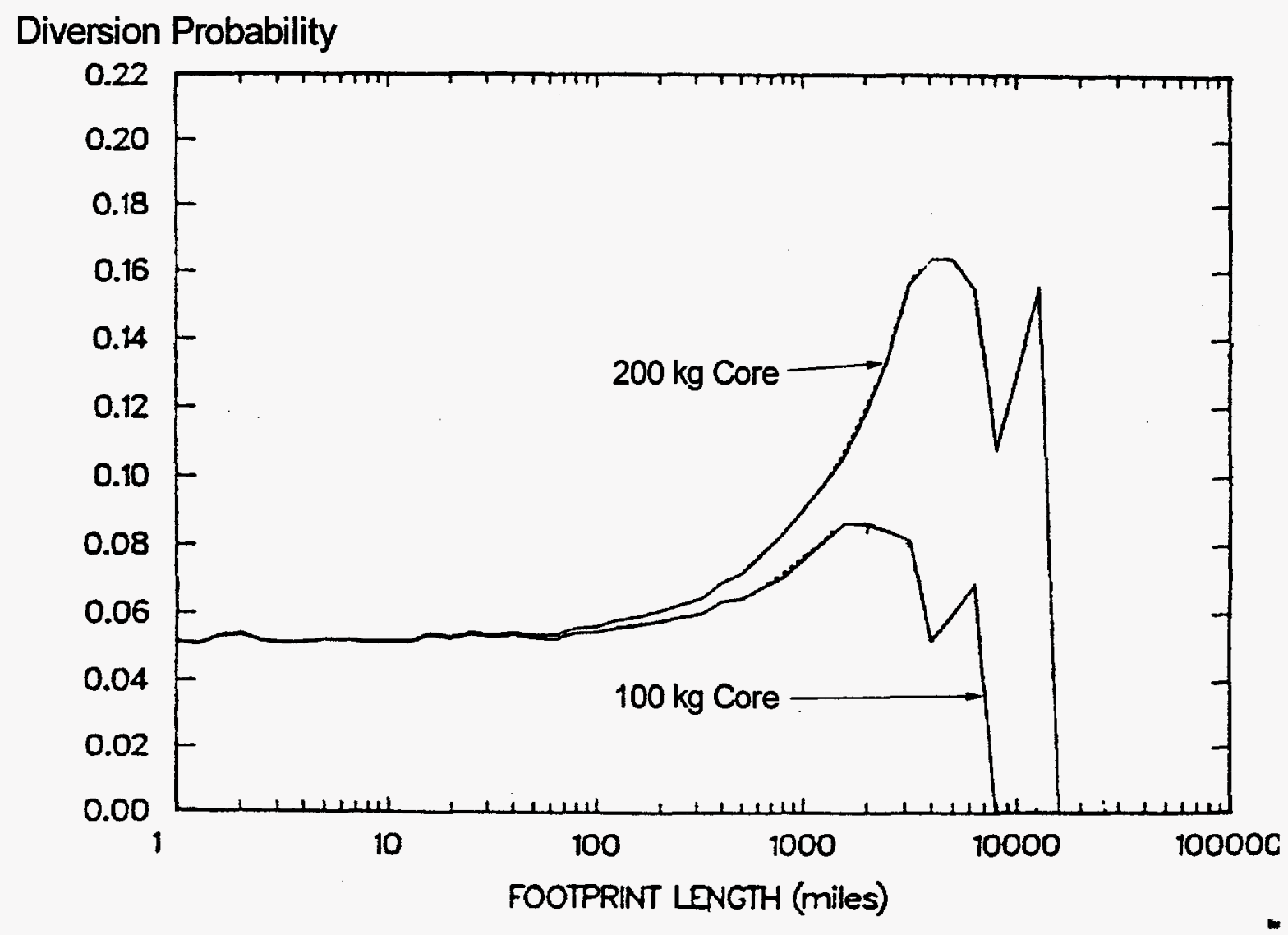

Figure 14. Los Alamos Calculations of SNM Diversion Probability

To better understand the TOPAZ II safeguards risks, a trajectory dispersion analysis was performed to estimate the length of the TOPAZ II ground footprint. The goal of the analysis was to bound the possible footprint lengths of SNM and determine if they were long enough to enter the region of increased diversion potential. 
The basic source of data for reentry conditions was a follow-up study of TOPAZ II reentry breakup, conducted by the Russian organization NP Energotech at the request of the NEPSTP safety team. This analysis modeled the destruction of the reactor system in greater detail. Table III summarizes the results of the NP Energotech study. The actual impact points of TOPAZ SNM will depend upon the point at which fuel pellets and/or rods separate from the reactor. This topic was not treated in the Energotech study. Therefore, impact points will be determined for three conditions: intact reactor, fuel rod, fuel pellet. Several release altitudes were used for the fuel rod and pellets. These altitudes are derived from the breakup events shown in Table III. It was judged that at those events the possibility of rod dispersal was greatest. 'For the case of fuel dispersal, the release altitudes were chosen to be $5 \mathrm{~km}$ lower than the fuel rod release.

Table III. TOPAZ II Reentry Breakup from NP Energotech Analysis

\begin{tabular}{|l|l|l|l|}
\hline Part Lost & $\begin{array}{l}\text { Mass Lost } \\
(\mathrm{kg})\end{array}$ & $\begin{array}{l}\text { Mass Left } \\
(\mathrm{kg})\end{array}$ & $\begin{array}{l}\text { Altitude } \\
(\mathrm{km})\end{array}$ \\
\hline Upper Plenum Lid & 3 & 952 & 82.9 \\
\hline Front Binding Tape & .3 & 951.7 & 76.3 \\
\hline Radiator Plates & 9. & 942.7 & 72.6 \\
\hline Upper Plenum Shell & 1. & 941.7 & 69.9 \\
\hline $\begin{array}{l}\text { Upper Plenum } \\
\text { Connections }\end{array}$ & 2.7 & 939. & 67.1 \\
\hline Radiator Tubes & 7. & 932. & 65.7 \\
\hline $\begin{array}{l}\text { Rear BindingTape, } \\
\text { Side Reflectors }\end{array}$ & 71. & 861. & 65. \\
\hline Radiator Frame & 260. & 601. & $61-64$. \\
\hline $\begin{array}{l}\text { Fuel Element Ends, } \\
\text { Front Tube Sheet }\end{array}$ & 21. & 580. & 58.7 \\
\hline Radiation Shield & 419. & 161. & 43. \\
\hline $\begin{array}{l}\text { Second Front Tube } \\
\text { Sheet }\end{array}$ & 0.7 & 160.3 & 42.7 \\
\hline Lower Plenum Shell & 0.64 & 159.6 & 40.7 \\
\hline $\begin{array}{l}\text { Dehydrogenation of } \\
\text { Moderator }\end{array}$ & 0. & 159.6 & 20. \\
\hline \hline
\end{tabular}

The impact points were calculated using the TSAP (Outka, 1989) point mass trajectory code. Aerodynamic coefficients were estimated from semi-empirical correlations. The TSAP results are shown in Figures 15 and 16. They show that the impact of fuel rods or pellets may take place over a range of approximately $250 \mathrm{~km}$ if dispersal starts at an altitude of $58.7 \mathrm{~km}$ and continues until core impact. 


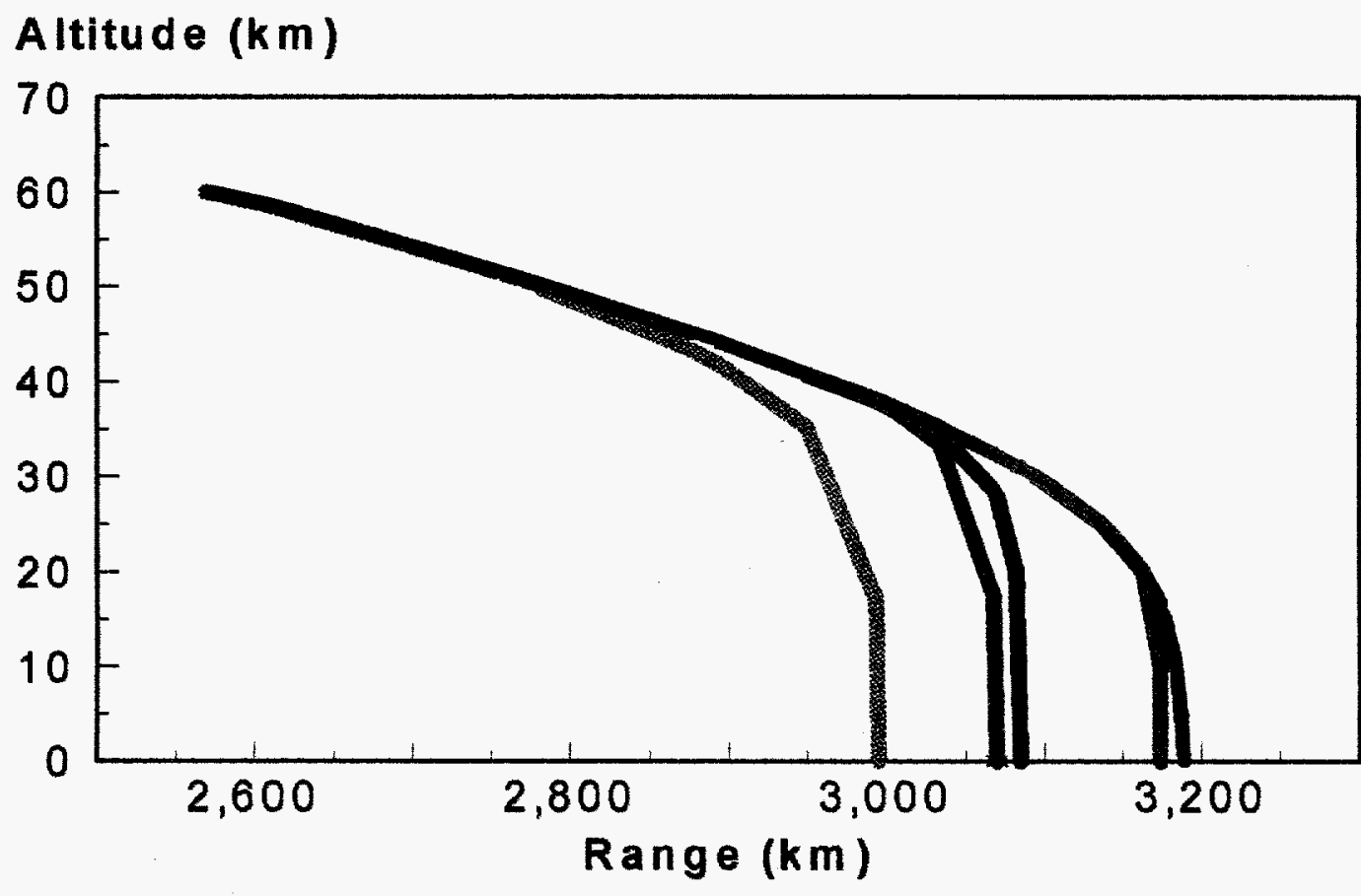

Intact $R x$

Fuel Rod $58 \mathrm{~km}$ -

Fuel Rod $43 \mathrm{~km}$ Fuel Rod $40.7 \mathrm{~km}$

Fuel Rod $20 \mathrm{~km}$

Figure 15. TOPAZ II Fuel Rod Impact Points

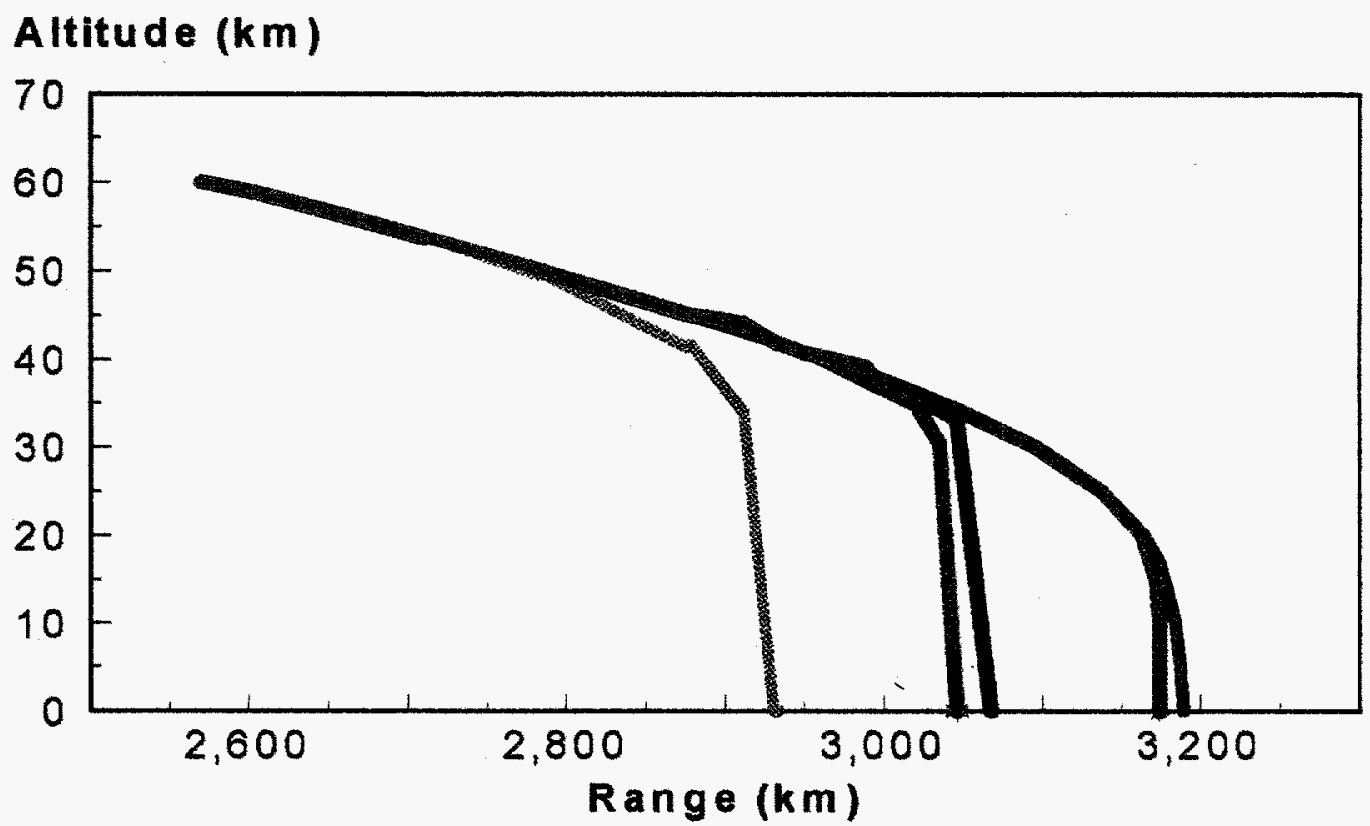

Intact $R x$

Fuel Pellet $53 \mathrm{~km}$ ( Fuel Pellet $38 \mathrm{~km}$

Fuel Pellet $35.7 \mathrm{~km}$

Fuel Pellet $15 \mathrm{~km}$

Figure 16. TOPAZ II Fuel Pellet Impact Points 
Therefore, (returning to Figure 14) it is evident that the probability of diversion of a significant quantity of TOPAZ II SNM will be the same low value of 5 percent regardless of whether the core reenters intact or is allowed to disperse over the worst case path length of $250 \mathrm{~km}$. Due to the nature of the diversion probability curves in Figure 14, this result holds for all reasonable choices of the significant quantity definition.

\section{Conclusions}

The analysis demonstrated that, for the risk factors considered, no risk reduction was obtained by forcing a radiologically cold TOPAZ II core to reenter intact. Consequently, the NEPSTP Safety Team reached a unanimous decision not to establish a core configuration functional safety requirement for cold reentry accidents. For any credible, radiologically hot reentry accidents, the functional safety requirement states that the core must either reenter intact or completely disperse at high altitude. This requirement becomes a rather mute point for TOPAZ II as design and operational constraints will preclude any credible radiologically hot reentry accidents. Therefore, the bottom line is that cold reentry accidents pose negligible radiological health risks and only minor safeguards risks, regardless of whether the core impacts the earth and remains intact or if all or part of the core disassembles during reentry or upon impact. 


\section{References}

Bennett G. L., (1989), "A Look at the Soviet Space Nuclear Power Program," IECEC89, August 1989.

Bost, D. S. (1985), "FY 1985 Surety Program Final Report," Rockwell International, Rocketdyne Division, RI/RD85-236, September 1985.

Connell, L. W. (1984), "A Model to Predict the Melting and Ablation of Reactive Metal Shrapnel," SAND83-1495, Sandia National Laboratories, Albuquerque, NM, May 1984.

DOE (1978), "Operation Morning Light - A Non-technical Summary of United States Participation," NV198, NTIS.

Eckerman, K. F. (1988), "Limiting Values of Radionuclide Intake and Air Concentration," EPA-520/1-88-020, Federal Guidance Report NO. 11, U.S. Environmental Protection Agency, Office of Radiation Programs, Washington D. C., 1988.

Elliot, R. D. (1963), "Aerospace Safety Reentry Analytical and Experimental Program SNAP 2 and 10A (Interim Report)," NAA-SR-8303, September 30, 1963.

Harty, R. B. (1985) SNAP-10 A Aerospace Nuclear Safety , A Good Foundation for the SP-100 Program, White Paper, Rockwell International Energy Systems Group, June 1985.

Koroteev, A. S., A. A. Gafarov, B. I. Bakhtin, A. V. Kosov, (1991), "On the Radiation Safety Studies of Space Nuclear Sources at the Scientific Research Institute of Thermal Processes," Space Nuclear Power Systems, 8th Symposium, January 1991.

Langham, W. W. (1967), "Biological Considerations of Nonnuclear Incidents Involving Nuclear Warheads," UCRL-50639, Lawrence Radiation Laboratory, Livermore, CA, June 1967.

Marshall, A. C. (1992), "Space Nuclear Power Safety Course Notes," Spring 1992.

Outka, D. E. (1990), "Users Manual for TSAP," SAND88-3158, Sandia National Laboratories, Albuquerque NM, July 1985.

Sullivan, H. (1985), "Interim SP-100 Reentry Dispersion Guideline," Los Alamos National Laboratory, Los Alamos, NM, April 1985.

Temme, M. I., Ed. (1985), "SP-100 Surety Evaluation," Nuclear Systems Technology Operation, General Electric Company, GEFR-00769, September 1985. 


\section{Distribution}

Gary L. Bennett

NASA Headquarters

Code RP

Washington, D. C. 20546

Stanley K. Borowski

NASA Lewis Research Center

Advanced Space Analysis Office

2100 Brookpark Road

Mail Stop AAC-2

Cleveland, $\mathrm{OH} 44135$

David Buden

Center for Nuclear Engineering and

Technology

INEL

P. O. Box 1625

Idaho Falls, ID 83415-2516

Wade P. Carroll

U. S. Department of Energy

NE-52

19901 Germantown Road

Germantown, MD 20874

John Clark

NASA Lewis Research Center

21000 Brookpark Road

Cleveland, $\mathrm{OH} 44135$

Mohammed El-Genk

Institute for Space Nuclear Power

Studies

Chemical and Nuclear Engineering

Department

University of New Mexico

Albuquerque, NM 87131

Eric F. Haskin

Institute for Space Nuclear Power Studies

Chemical and Nuclear Engineering Department

University of New Mexico

Albuquerque, NM 87131
J. F. Janni, Chief Scientist

Phillips Laboratory, PLCO

3550 Aberdeen Avenue S. E.

KAFB, NM 87117-5776

Patrick J. McDaniel

Phillips Laboratory/WSA

Kirtland AFB, NM 87117-6008

James R. Powell

Building 701

Brookhaven National Laboratory

Upton, NY 11973

$\begin{array}{ll}\text { MS0826 } & \text { D. L. Potter, } 1553 \\ \text { MS0826 } & \text { C. C. Wong, 1553 } \\ \text { MS0149 } & \text { C. P. Robinson, } 4000 \\ \text { MS0455 } & \text { G. R. Otey, 4100 } \\ \text { MS0421 } & \text { W. C. Hines, } 4101 \\ \text { MS0415 } & \text { C. B. Layne, } 4102 \\ \text { MS0415 } & \text { G. S. Brown, 4111 } \\ \text { MS0419 } & \text { C. C. Carson, 4112 } \\ \text { MS0417 } & \text { W. H. Ling, 4113 } \\ \text { MS0423 } & \text { J. W. Keizur, 4114 } \\ \text { MS0425 } & \text { R. R. Preston, 4115 } \\ \text { MS0736 } & \text { N. R. Ortiz, 6400 } \\ \text { MS1176 } & \text { J. H. Lee, 6474 } \\ \text { MS1176 } & \text { A. C. Marshall, 6474 } \\ \text { MS1176 } & \text { F. V. Thome, 6474 } \\ \text { MS1143 } & \text { J. K. Rice, 6500 } \\ \text { MS1144 } & \text { J. V. Walker, 6501 } \\ \text { MS1144 } & \text { R. X. Lenard, 6501 } \\ \text { MS1175 } & \text { G. C. Allen, 6513 } \\ \text { MS1175 } & \text { G. F. Polansky, 6513 } \\ \text { MS0425 } & \text { L. W. Connell, 4115 (25) } \\ \text { MS9018 } & \text { Central Technical Files, } \\ & \text { 8523-2 (1) } \\ \text { MS0899 } & \text { Technical Library, } \\ & \text { 7141 (5) } \\ \text { MS0619 } & \text { Technical Publications, } \\ & 7151 \text { (1) } \\ \text { MS0100 } & \text { Document Processing for } \\ & \text { DOE/OSTI, 7613-2 (10) }\end{array}$

